\title{
Variations
}

Variations

Revue internationale de théorie critique

$19 \mid 2016$

Critique des humanités numériques

\section{Critique et humanités numériques}

Pour une approche matérialiste de l'« immatériel »

Fabien Granjon et Christophe Magis

\section{(2) OpenEdition}

Journals

Édition électronique

URL : http://journals.openedition.org/variations/748

DOI : $10.4000 /$ variations. 748

ISSN : 1968-3960

Éditeur

Les amis de Variations

Référence électronique

Fabien Granjon et Christophe Magis, "Critique et humanités numériques », Variations [En ligne],

19 | 2016, mis en ligne le 06 avril 2016, consulté le 30 avril 2019. URL : http://journals.openedition.org/ variations/748; DOI : 10.4000/variations.748

Ce document a été généré automatiquement le 30 avril 2019.

Les ami•e•s de Variations 


\title{
Critique et humanités numériques
}

\author{
Pour une approche matérialiste de l'« immatériel »
}

Fabien Granjon et Christophe Magis

«-Quelle est votre spécialité McGarrigle? $-\mathrm{Heu}, \mathrm{j}$ 'ai fait ma recherche sur Shakespeare et T.S.

Eliot, dit Persse [...]

-C'est un sujet idéal à informatiser, poursuivit

Dempsey, vous n'auriez qu'une chose à faire, mettre les textes sur bandes et l'ordinateur vous donnerait la liste de tous les mots et de toutes les constructions syntaxiques que les deux écrivains ont en commun. Vous pourriez ainsi quantifier de manière précise l'influence de Shakespeare sur T.S.

Eliot.

-Mais ce n'est pas le sujet de mon mémoire, dit

Persse. Il porte sur l'influence de T.S. Eliot sur

Shakespeare. »

David Lodge, Un tout petit monde.

« Beware of the great diminishers: they will reduce

anything in digital humanities and preface our

work with “just" (it's just a tool; it's just an archive; it's just pedagogy). They have never built

software, parsed code, created a database, or designed a user interface. They just write articles

and books. »

A Digital Humanities Manifesto, 2008.

1 La présente contribution souhaite interroger la prétention des Humanités Numériques $\left(\mathrm{HN}^{1}\right)$ à redistribuer les cartes des sciences humaines et sociales (SHS) ${ }^{2}$. Plus précisément, nous voudrions initier une réflexion inspirée du rapport que Lucien Goldmann suggérait d'avoir vis-à-vis des sciences sociales : commencer à penser «la signification» des 
humanités numériques, c'est-à-dire "la nature de leur lien avec la réalité sociale contemporaine, le sens de leur action à l'intérieur de cette réalité et les cadres sociaux qui pourraient favoriser ou au contraire diminuer leur valeur positive en tant qu'instruments de connaissance [et d'émancipation] » (1966: 16). Dans cette perspective, nous ambitionnons de passer les $\mathrm{HN}$ au crible de la critique matérialiste (d'un structuralisme génétique aurait proposé Goldmann). Par là, nous ne désirons pas les "prendre à revers ", mais bien les prendre au sérieux en revenant sur le programme le plus élevé que certains souhaitent leur attribuer: "rouvrir un champ d'interrogations sur les pratiques de recherche en sciences humaines et sociales et les épistémologies qui les fondent, et aussi [...] sur le rapport qu'elles établissent aux autres disciplines scientifiques, et même avec l'ensemble de la société » (Mounier, 2004: 96). Nous essaierons notamment de montrer que, depuis leur intérêt pour le numérique, les HN posent de nouveau un certain nombre de questionnements qui entrent en résonance forte avec le principium de la pensée critique (Granjon, 2013) dont certains attendus ont d'ores et déjà inspiré d'intéressants travaux sur la "culture numérique» et ses implications économiques, sociales et politiques, que ces derniers se réclament ou pas des humanités numériques (Silver, Massanari, 2006; Kroker, Kroker, 2008 ; Citton, 2015 ; Berry, 2015 ; Fuchs, 2014, Revue Triple-C, etc.).

Dans cette optique, nous nous proposons d'aborder le thème du brouillage des frontières scientifiques que les humanités numériques érigent en principe central, ainsi que la question du big data qui les occupent tout autant. Nous envisagerons ces thématiques comme devant être appréhendées comme prolongements et transpositions d'une vision matérialiste de l'interdisciplinarité et de la totalité. Nous nous attacherons, ensuite, à penser l'exacerbation, par les HN, de l'importance du «faire " (une mise en avant du « hacking ") comme une vision pragmatiste des rapports entre théorie et pratique. Enfin, nous verrons comment les nouvelles manières de faire science, proposées par les HN, réinvestissent à leur manière la critique des catégories de production et de propriété. Notre réflexion vise ainsi à évaluer les potentiels de développement plus radicaux, en rapport avec les attendus d'une critique matérialiste. Elle est également une invite à faire vivre ce que d'aucuns ont appelé "the dark side of digital humanities ${ }^{3}$ " (Grusin, 2013; Differences, 2014), c'est-à-dire à pousser la nécessité du développement d'humanités numériques critiques ${ }^{4}$.

\title{
Interdisciplinarité et big data : figures de la totalité
}

\author{
« Disciplines and disciplinary traditions can be \\ wellsprings of quality, depth, and rigor. They can \\ also be bastions of small thinking, clerical \\ privilege, and intellectual policing. » \\ The Digital Humanities Manifesto 2.0, 2009.
}

3 Les humanités numériques sont souvent présentées comme la suite des humanities computing (elles-mêmes extension des literacy computing: Burnard, 2012 ; Kirschenbaum, 2010; Schreibman et al., 2004), c'est-à-dire comme un moment de réflexivité des humanités quant à l'utilisation de plus en plus prégnante des technologies informatiques dans les études littéraires et, plus largement, celles des SHS. Les importations de techniques quantitatives issues des études informatiques ont par exemple donné lieu à des réflexions critiques sur les méthodologies des SHS (Hayles, 2012 ; Hirsch, 2012; Manovitch, 2012), 
réflexions que les HN poursuivent aujourd'hui en s'interrogeant sur la manière de faire science et de penser certains fondements épistémologiques (Laue, 2004 ; Burdick et al., 2012). Dans cet ensemble réflexif, l'émergence des humanités numériques semble coupler à une première nécessité : celle de déplacer les frontières disciplinaires, de provoquer « une radicale modification du paysage intellectuel » (Doueihi, 2013:12) et donc de ne pas se contenter des «logiques administratives exclusivistes» (l'expression est de Jean-Marie Brohm). Les HN auraient vocation à proposer des formes innovantes de mise en cycle du savoir fondées sur de nouvelles alliances entre certaines sciences dites "dures" (biologie, mathématiques, informatique, etc.) et les sciences humaines et sociales, considérées de la manière la plus extensive qui soit (littérature, arts, histoire, anthropologie, sociologies, etc.) : «Les digital humanities désignent une transdiscipline, porteuse des méthodes, des dispositifs et des perspectives heuristiques liés au numérique dans le domaine des Sciences humaines et sociales » (Dacos, 2011). Elles feraient, nous diton, « entrer en dialogue toutes les disciplines qui les composent » (Mounier, 2014a : 99), « évoluer le travail collectif» (Dacos, 2011) et offriraient, à la faveur de la "révolution numérique ", des opportunités pour réviser la division sociale du travail scientifique. Dans l'idée d'une démarche complémentariste susceptible de rétablir des ordres relationnels indûment découpés, les HN enjoignent donc ceux qui souhaitent y prendre part, de prendre au sérieux l'environnement technique dont elles soulignent qu'il participe de la conduite politique (historique) du social. Et l'on peut en effet penser qu'étudier le numérique dans ses diverses dimensions (production, implémentation, appropriation, etc.) conduit sans doute à ce que chacune des sciences susceptibles de s'y intéresser rabatte quelque peu sa prétention à détenir, seule, la vérité sur le digital turn.

4 À l'instar du mouvement de fond accompagnant le développement des sociétés capitalistes avancées, le champ scientifique est effectivement pris, lui aussi, dans une dynamique de différenciation disciplinaire et de spécialisation de la recherche, recherche qui s'intéresse bien souvent à des microcosmes, des faits ou des objets sociaux parfois très spécifiques et la plupart du temps avec une grande précision, mais qui peine néanmoins à les resituer au sein de logiques sociales plus générales qui pourtant les produisent et auxquelles ils participent. Force est de constater qu'au sein des SHS, le tout tend ainsi plutôt à être conçu comme la somme des différentes parties étudiées par les différentes disciplines et sous-disciplines. "Mais par-là se perd le lien des différentes parties entre elles et la relation des parties au tout. La totalité n'est plus une unité, elle n'est plus que la somme des domaines particuliers du Savoir. [...] [Et] la dislocation n'atteint pas seulement le lien des différentes sciences entre elles, mais aussi leurs sphères intérieures mêmes qui se morcellent de plus en plus en différents domaines partiels» (Jakubowski, 1971: 162-163). On pourrait même aller jusqu'à faire l'hypothèse que cette difficulté va croissant et que les sciences humaines et sociales auraient de plus en plus de mal à fournir des explications un tant soit peu globales des sociétés. Les intérêts de connaissance se fragmentent et conduisent au final à une balkanisation de la recherche, laquelle contribue, selon Bernard Lahire, à une perte « du sens des totalités sociales et des liens d'interdépendance qui existent entre des domaines différents de la pratique » (2012: 322).

«La recherche anthropologique moderne établit la complexité de l'homme comme hypothèse de base, reflétant ainsi l'esprit de la méthode scientifique et du nombre croissant de disciplines qui étudient l'homme. L'homme est un être compliqué qui ne peut être expliqué par une quelconque formule métaphysique. Chacun de ses intérêts particuliers est érigé en sujet d'une discipline scientifique indépendante, 
afin d'être analysé avec exactitude. Les diverses sciences anthropologiques spécialisées ont assemblé une masse énorme de matériaux, et ont mis en avant des découvertes inestimables sur l'homme en tant qu'être biologique, social, etc. Pourtant, en dépit de ces prouesses scientifiques, l'homme n'a jamais autant été un problème qu'aujourd'hui. Cette contradiction est due à une conception impropre du rôle de l'anthropologie scientifique. Les différentes sciences humaines s'occupent de l'un ou de l'autre des aspects particuliers de l'homme. Lorsqu'elles expliquent leurs observations de façon systématique, ces sciences procèdent de leur propre point de vue afin de développer une conception de l'homme en tant que tout. Le problème qu'elles se posent tient dans la question Qu'est-ce que l'homme? Leurs réponses viennent s'ajouter à une quantité déprimante de définitions, puisque chacune s'autorise à un champ toujours plus large dans l'établissement des caractéristiques fondamentales de l'homme. Il est vrai que l'homme est un être vivant qui produit des outils mais il est également vrai de dire que c'est un être vivant qui utilise des symboles, qui a conscience de sa propre mortalité, qui est capable de dire non, que c'est un être social, etc. Une définition ne peut contester les hypothèses de l'autre, car chaque aspect particulier de l'homme est isolé et aucun d'entre eux n'est capable, de son point de vue particulier, de fournir une notion de l'homme en tant que tout, concrètement et dans sa totalité » (Kosik, $2003: 34-35)$.

5 L'exigence d'interdisciplinarité n'a toutefois pas attendu l'émergence des humanités numériques pour s'exprimer et répond notamment, sous les auspices de la critique, à la nécessité de saisir dialectiquement le singulier, le particulier et le général (Granjon, 2014a). Mais dégagée de sa charge critique, elle tend souvent à se présenter comme « un slogan pour inciter les spécialistes à ne plus borner leur domaine, à travailler aux marges, voire à circuler sauvagement dans l'ensemble de l'univers des savoirs » (Vinck, 2000 : 56). Cette vision d'une pensée enfin débridée, libre d'aller et venir entre les différents pôles de la production scientifique se justifierait, selon Dominique Vinck, par différents types de nécessité :

6 - D’une part, la complexité des objets étudiés : il y aurait ainsi des objets "par nature interdisciplinaires " qui renverraient à «des causalités multiples». De facto, les HN considèrent parfois que le numérique relève d'un «fait social total» (Mauss, 1973) qui, traversant les sociétés capitalistes avancées de part en part, constitue la dynamique civilisationnelle (historique) de fond, laquelle nécessite une approche plurielle. Mais penser comme une addition ex post de regards de spécialistes rendus nécessaires par la supposée « complexité » d'un objet donné ne contribue-t-il pas à maintenir le statu quo que l'interdisciplinarité affirme vouloir ébranler, et ce à deux niveaux : à la fois à propos du morcellement du savoir en monades disciplinaires ultra-spécialisées - qu'elle tendrait paradoxalement à renforcer -, ainsi qu'au niveau de la hiérarchie entre les disciplines, qui pousse in fine à la subsomption des catégories d'analyse à celles de l'économie dominante ou de la sociologie positiviste et à leur aspect factice et mystifiant? Depuis une perspective critique, l'interdisciplinarité ne se justifie pas tant par la complexité singulière des objets appréhendés, que précisément par le regard critique qui vise à penser la totalité sociale et ses différents moments. L'interdisciplinarité apparaît donc comme une nécessité émanant pour l'essentiel de la volonté de totalisation et de relier les phénomènes étudiés à des ordres sociaux qui en conditionnent l'existence.

7 - D'autre part, l'indexation aux demandes technologiques, économiques et sociales : « Dans un contexte socio-économique où le souci est de mettre la science au service du développement technologique et industriel, on comprend que les gestionnaires de la recherche ragent devant la multiplicité des chapelles scientifiques et qu'ils plaident pour 
l'interdisciplinarité » (Vinck, 2000: 57). C'est par exemple sous couvert de cette conception que, dans le plan d'action 2015 de l'Agence Nationale de la Recherche (en lien direct avec l'Agenda stratégique " France Europe 2020 »), l'interdisciplinarité est envisagée comme un levier de la compétitivité et aurait partie liée avec le "développement de la formation et des infrastructures numériques» (Hirsch, 2012). L'interdisciplinarité est ici conçue comme une méthode scientifique à mettre notamment au service du développement de l'« e-éducation ", des fameux Moocs (Massive Open Online Courses - l'Unesco parle pour sa part, d'Open Educational Resources - Mahony et al., 2012) et du programme "France université numérique ", au même titre que le numérique serait, pour sa part, un outil technologique « au service de toutes les sciences» (Spiro, 2012a). Interdisciplinarité et numérique seraient ainsi liés dans un même combat qui aura notamment à passer, nous dit-on, par le renforcement par la France de ses «einfrastructures ", de sa "puissance de calcul » et par le déploiement du cloud computing pour les données massives de la recherche, le tout à des fins de transfert des compétences aux entreprises. Bref, il s'agit de faire ce qu'aux États-Unis le Bureau des Humanités numériques du National Endowment for Humanities (NEH) a développé depuis 2008: un programme de développement des infrastructures numériques et du big data (Manovich, 2012 - notamment via le concours « Digging into Data Challenge »). Ainsi envisagée, dans une démarche de problem solving, l'interdisciplinarité vise surtout à répondre aux demandes sociales portées par les innovateurs de technologies numériques et la mythologique "classe créative " (universitaires, artistes et/ou entrepreneurs) désireux de s'appuyer sur une production de connaissance ouverte leur permettant d'investir les contextes d'applications les plus rentables (économiquement, socialement, etc.) de leur domaine. Cette marginalité épistémologique allant à l'encontre de l'institutionnalisation disciplinaire, nous semble néanmoins problématique dans la mesure où, en cette justification particulière, elle rejette bien souvent la « condition scolastique » au nom des freins que cette dernière constituerait vis-à-vis d'une dynamique entrepreneuriale et libérale (prise de risque, mobilité, projets) qu'il s'agirait de prendre pour modèle. Aussi, induit-elle une autre forme d'hétéronomie, celle-là même qui faisait déjà dire à Marx que la valeur d'usage de l'économie politique était découplée de sa propre activité et se plaçait sous la coupe d'une dépendance externe travaillée par les intérêts du Capital. Celle aussi qui lui faisait par ailleurs considérer que le matérialisme historique était un point de vue de classe qui avait vocation à se saisir théoriquement de la réalité sociale pour la changer pratiquement. Celle encore qui se trouve au principe de la distinction que Max Horkheimer (1974) établit entre théorie traditionnelle et théorie critique, cette dernière devant porter haut, contre la "science bourgeoise", les fondements d'une science dialectique ouverte et émancipatoire (Voirol, 2012 ; Christ, 2012).

- Enfin, la créativité scientifique-conquête indisciplinée qui n'aurait que faire des dogmes académiques et arpenterait plusieurs territoires, préférant se tenir aux marges et aux frontières et se donner ainsi la possibilité de «défricher de nouvelles terres ", à l'écart des normes et des paradigmes les plus prégnants. Certains animateurs des humanités numériques (les plus « scientists») recommandent une ouverture interdisciplinaire dont le principal objectif serait de remettre en cause les clôtures scientifiques au nom d'une science qui se doit précisément d'être plus ouverte et se rapprocher d'« une logique de la création [qui] ne reconnaît [...] ni ne ratifie les frontières au moment même où elle les franchit; elle les démantèle et les dissout, pour dessiner de nouveaux types d'appartenances intellectuelle » (de Lagasnerie, 2011: 162). Il s'agirait notamment d'«inventer une nouvelle manière "d'encyclopéder les savoirs", afin de penser les 
enchevêtrements de relations, d'écritures, de concepts, de points de vue et de traductions » (Noyer, $2010: 189$ ).

Cet intérêt à se jouer des frontières académiques a tenté d'être relevé par certaines disciplines qui revendiquent selon les contextes : soit une pluridisciplinarité consistant «à juxtaposer des points de vue relevant de disciplines distinctes » en mobilisant des méthodes identiques ou complémentaires sur des objets communs; soit une interdisciplinarité qui "suppose un dialogue, un échange ou une confrontation entre plusieurs disciplines» (Vinck, 2000: 61-62), généralement par importations et traductions de problématiques et/ou de concepts. En ce cas, «le spécialiste essaye, du point de vue de sa science particulière et avec les méthodes qui lui sont propres, d'embrasser d'autres domaines et de réaliser ainsi l'unité de la connaissance par l'élargissement de sa propre sphère " (Jakubowski, 1971: 161) ; soit enfin une transdisciplinarité, laquelle est envisagée comme un dépassement des disciplines. Il nous semble que les humanités numériques vont rarement jusqu'à ce dernier degré d'exigence, en tout cas discutent rarement cette nécessité et, de facto, les disciplines restent considérées comme " point de départ à partir duquel on s'interroge sur les interactions entre disciplines ou sur la nécessité de dépasser la discipline " (Vinck, 2000: 63). Aussi, Melissa Terras considère plutôt les HN comme restant marquées par la spécialisation, une espèce de "grande église [où] les expériences et les connaissances des individus sont si faiblement liées que le mot de "pairs" donne souvent une mauvaise idée de la pertinence de l'applicabilité d'un domaine de recherche spécifique donné » (Terras, 2012).

10 La pensée critique, à commencer par le matérialisme marxien dont Karl Korsh affirmait qu'il ne «ne se laisse ranger dans aucun des tiroirs habituels du système des sciences bourgeoises » (1979:7) et dont Jean-Marie Brohm note qu'il est "une théorie-praxis unitaire, globale, qui s'approprie la totalité de la réalité historico-sociale sans se fragmenter dans les différentes sciences sectorielles réifiées » (1974: 38), a fait de la transdisciplinarité un impératif. Pour la critique, il s'agit en effet de se donner les moyens de penser les "phénomènes sociaux » comme en mouvement. Le matérialisme critique " suppose d'abandonner le "point de vue du laboratoire", intellectuellement rassurant, au profit du "point de vue de la totalité" [...] qui implique de tenir compte de toutes les dimensions de la réalité, tout en abordant cette dernière dans la perspective déterminée [d'un problème particulier] - raison pour laquelle sa visée est totalisante sans qu'il recherche pour autant l'exhaustivité » (Berlan, 2012 : 48-49). Il s'agit donc de réaffirmer l'exigence d'avoir à penser la totalité sociale et ses différents moments (notamment technologique), ce qui nécessite, répétons-le, de comprendre les faits sociaux comme des réalités dynamiques structurées et structurantes. La variété des SHS peut alors se concevoir comme une base à partir de laquelle il s'agit de décomposer un «tout » en unités d'analyse pour l'appréhender de nouveau comme un «tout», mais un "tout» recomposé par une médiation théorique (critique) qui l'éclaire sous un nouveau jour pour lui donner du sens en faisant tenir dans une totalité ce que la facticité empiriste et positiviste déli(t)e, disjoint et naturalise. À cette aune, on peut par exemple envisager les faits sociotechniques comme historiquement ancrés au sein d'un "général », en adoptant un point de vue qui les recontextualise dans une structure sociale globale et les appréhende comme des singularités porteuses de cette structure du tout. Autrement dit, la transdisciplinarité critique permet, entre autres choses, de saisir le numérique comme participant à des totalités structurées, intérêt que les humanités numériques n'ont pas nécessairement comme horizon (cf. infra). 
11 L'exigence de totalisation est certes présente au sein des humanités numériques mais elle est envisagée, la plupart du temps, sous l'angle du big data (Boyd, Crawford, 2011) et de la tentation quantophrénique qui l'accompagne parfois. Aussi, l'interdisciplinarité lui correspondant en reste bien souvent à la "quête d'un universalisme » d'ordre mathématique qui serait au fondement des sciences occidentales (Rieder, Röhle, 2012). Nous sommes alors pour le moins éloignés des attendus du principe de totalité de la critique: "En considérant le fait humain comme un fait mobilisé dans une base de données, ces sciences adoptent de fait un positivisme naïf où le fait collecté est la seule positivité à interroger et d'où il faut partir pour appliquer les modèles de construction scientifique» (Bachimont, 2014: 77). Aussi, comme le note Doueihi, la recherche de la totalisation portée par le tournant numérique se traduit encore trop souvent par « une quête d'exhaustivité qui anime la survalorisation des données » $(2013: 20)$ et il semble un peu court de considérer que " le plus grand espoir pour le renouvellement de nos traditions théoriques communes dans la recherche en sciences humaines, et peut-être la seule voie possible, est d'utiliser le stockage numérique massif de données " (Schmidt, 2011). La totalisation "made in $D H »$ se couple alors à un attachement pour les algorithmes, les instruments de data mining, les graphes de réseaux et autres représentations graphiques novatrices (e.g. via des cartes de Kohonen) qui, certes, permettent de rendre visible d'une nouvelle manière des éléments de la réalité sociale, mais dont la sophistication ne garantit en rien l'heuristique. Si les outils numériques « permettent d'étudier l'ensemble des données comme un tout » (Bachimont, 2014 : 70), le tout en question ressemble encore trop fréquemment à une totalité abstraite rassemblant des données qui peinent à se référer, pour paraphraser Marx, à des réalités concrètes et "vraiment vivantes ». Autrement dit, la connaissance de la totalité façon « big data + data mining» est-elle aussi connaissance d'un sujet social ? Rien n'est moins sûr et l'on flirte bien souvent avec une forme nouvelle de " fétichisme du texte ", ici plutôt fétichisme des données (data). Lev Manovich précise par exemple qu'il est possible de «faire des recherches intéressantes en analysant plus grand nombre de tweets, de photos Facebook, de vidéos YouTube, ou tout autre site de réseau social, mais [que] nous devons juste garder à l'esprit que toutes ces données ne sont pas une fenêtre transparente sur l'imagination, les intentions, les motifs, les opinions et les idées des personnes» (2012: 466). Les cartographies du web nous semblent être l'exemple type de formes de totalisation qui peuvent être impressionnantes dans leur façon de rassembler à l'aide d'une même représentation des données en établissant des liens entre elles, mais sans être en mesure, la plupart du temps, de les raccrocher à une forme de totalisation autre que simplement descriptive, voire de rencontrer quelque difficulté à développer les sémantiques de lecture de ces liens (Ouakrat, Mésengeau, 2016). Tout se passe comme si ces cartes inédites devenaient suffisantes pour décrire, expliquer, comprendre et juger des « territoires sociaux » qu'elles représentent. Tel n'est évidemment pas le cas et cela donne notamment lieu à des tensions entre "ceux qui suggèrent que les humanités numériques devraient toujours relever d'un "faire" (que ce soit la constitution d'archives, la fabrication d'outils ou de nouvelles méthodes numériques) et ceux qui suggèrent qu'elles devraient porter davantage sur l'interprétation des données » (Fitzpatrick, 2011b). 


\title{
Pratique et théorie : philosophie pragmatique et matérialité technologique
}

\author{
«In practice, this probably means digital \\ humanists can keep calm and carry on, with \\ greater tolerance for the occasional French name \\ tossed into the discussion. " \\ Benjamin M. Schmidt, « Theory First », 2011.
}

Les humanités numériques décrivent, selon Pierre Mounier s'appuyant sur Adema Janneke (2013) « une praxis critique d'un espace ouvert par la tension entre les paradigmes de l'informatique et des sciences humaines " (2014: 108). Sur cette base, elles conduisent, comme nous l'avons vu, à une critique de la balkanisation croissante des sciences, de la condition scolastique la plus élitaire, de la fragmentation des savoirs, de leur confinement par spécialisation, de leur réservation à l'espace académique et de leur restitution par bribes. À l'évidence, les humanités numériques souhaiteraient faire fond sur des valeurs pragmatiques, d'utilité, de sensibilisation du public et du dépassement d'une science assujettissante et réservée (Schmidt, 2011). Aussi, à leur manière, elles réaffirment, nous semble-t-il, la nécessité de retisser des relations entre le savoir et l'action, de relier le domaine de la connaissance et celui de l'agir : « La dichotomie entre le royaume manuel de la pratique et le royaume mental de la pensée a toujours été trompeuse. Aujourd'hui, le vieux débat théorie/pratique n'a plus lieu d'être. La connaissance suppose des formes multiples » (Schnapp et al., 2009). Natalia Cecire (2011) considère, pour sa part, que les HN doivent être davantage préoccupées par la méthode que par la théorie que d'aucuns considèrent d'ailleurs comme un paravent de l'idéologie (Scheinfeldt, 2010 ; 2012). Le débat se concentre notamment autour de la préférence qui doit être donnée au "hack» (faire - epistemology of doing, making, building) plutôt qu'au «yack» (baratin théorique Murray-John, 2011 ; Berra, 2012 ; Ramsay, 2011), c'est-à-dire en des termes plus courants, à la prévalence de la pratique sur la théorie et plus particulièrement, en l'occurrence, de l'empirie sur la théorie. L'invitation est claire: il s'agit de «mettre les mains dans le cambouis" (hands-on, getting your hands dirty). Mais travailler sur des matériaux empiriques (des données - B-A-BA des sciences sociales), construire des logiciels, des interfaces, présenter des cartographies, etc., ne saurait être considéré comme relevant d'un répertoire de pratiques s'opposant à « la théorie ». Les plus conscients des dangers que représente ce "grand partage" (positivisme et empirisme vs. idéalisme et théoricisme - Fitzpatrick, 2012) en appellent par exemple à la constitution d'une " praxis critique " (Adema, 2012 ; Schmidt, 2011), d'une "pratique technico-critique» (Dieter, 2014), ou encore d'une "ingénierie critique " (Oliver et al., 2011) couplant littéracie numérique et compétences critiques (au sens ici de «théoriques »). L'invite est celle d'une «pratique critique articulée à la possibilité concrète de hacker, pirater, bidouiller, bloquer et reconfigurer de nouveaux cheminements et de nouveaux possibles » (Berry, 2015 : xx). Sous les conditions de la critique, théorie et pratique forment en effet un couplage structurel (e.g. Hooks, 1994). L'unité de la pensée et de l'action, «le mouvement de la réalité dans la pensée et le mouvement de la pensée dans la réalité » (Brohm, 2003 : 30) se présente comme une base de la dialectique matérialiste pour laquelle la coupure entre la théorie et la praxis est une aporie. La praxis ne s'oppose pas au mouvement de la pensée, elle lui est fondamentalement liée et peut donc prendre des formes différentes 
selon le type de connaissance qui est mobilisée. Karel Kosik (1988) évoque ainsi l'existence d'une "praxis quotidienne " (« d'ajustement » pourrait-on dire) fondée sur une connaissance routinière, de sens commun, qui s'oppose en tout point à la praxis de la critique qui, elle, se veut libératoire et émancipatrice. Les humanités numériques devraient ainsi, d'une part, se méfier du rejet du théorique au nom de la pratique, ce qui, du point de vue de la critique est une antinomie, et devrait, d'autre part, s'interroger plus avant sur ce que cette opposition factice (idéologique) qui semble teintée de bon sens (ce qui fait sa force) conduit à développer comme type de vision de la réalité en vue de l'action, mais aussi la manière dont cette action fait retour sur la pensée.

13 Aussi s'agit-il pour les $\mathrm{HN}$ d'effectuer un effort de réflexivité quant à cette volonté affirmée d'être d'abord dans le "hack» et dont on peut douter qu'elle favorise une distance consciente avec le monde tel qu'il va, tout comme du rôle qu'y joue la numérisation du signe. Assurément, elle ne favorise en rien l'émergence d'humanités numériques de portée critique qui pourraient se constituer en «une force politique perturbatrice " (a disruptive political force - Gold, 2004: x) que certains DHers (i.e. les universitaires se réclamant des Digital Humanities) semblent pourtant appeler de leurs vœux. « Hacker » peut se résumer à « bidouiller » à des fins utilitaires, mais cela peut aussi être une action transformatrice constituant une unité entre critique théorique et critique sociale. L'appel de Cathy Davidson (2009) à ses collègues DHers à utiliser les technologies numériques pour s'engager dans un combat politique (en l'occurrence contre le régime des mollahs en Iran) touche à l'évidence à cette volonté d'engagement (Losh, 2012 - cf. infra). Plus généralement encore, être critique revient à «être capable de recul par rapport à soi-même et par rapport au monde dans lequel nous vivons, de pouvoir sortir du présent et de l'insertion dans ce présent dans la totalité historique pour pouvoir distinguer dans ce présent le particulier du général, le contingent du réel, le barbare de l'humain, l'authentique et l'inauthentique » (Kosik, 2003 : 72). Aussi, la critique théorique invite à s'interroger sur les modes de production des connaissances, sur les rapports sociaux dans lesquels ils s'insèrent, sur le travail scientifique comme composante de la division intellectuelle du travail, ainsi que sur ses conditions de réception sociale. Les HN, ou à tout le moins une partie d'entre elles, semblent donner quelque crédit à cette exigence et ne sauraient donc faire l'impasse sur le fait d'avoir à s'interroger sur les conséquences de l'adhésion à ce programme depuis un intérêt central pour le numérique.

Les humanités numériques se posent donc, pour les plus réflexives, la question de leur responsabilité sociale (Bianco, 2012 ; Cecire, 2011) et de l'engagement des chercheurs qui s'en réclament, bien que d'aucuns leur reproche précisément leur manque d'engagement politique concret, par contraste, par exemple, avec le champ d'étude des «nouveaux médias » qui semble plus proche des milieux hacktivistes (Liu, 2012; Losh, 2012 ; Raley, 2014). Sans doute est-ce parce qu'elles fondent leurs « valeurs » les plus centrales sur des principes qui ne ressemblent que peu à celles d'un travail du négatif typique de la critique sociale la plus traditionnelle, mais puisent davantage à des formes d'action collective plus directement liées à des communautés et à des sujets dont les revendications tournent autour de la critique culturelle et de la politisation de la technique: «Nous devons développer des langages communs qui lient l'étude des codes et de la culture. Nous devons historiciser et politiser les études sur le code » (McPherson, 2012 : 153). Lisa Spiro (2012b), Roger Whiston (2012) ou Tom Scheinfeldt $(2009 ; 2010)$ considèrent par exemple que le « logiciel » éthique des HN promeut des valeurs telles que l'ouverture (open access, open data, etc.), la collaboration, la participation, la collégialité, la solidarité, 
l'horizontalité, la diversité, l'humilité, ou encore l'expérimentation. Cette dernière apparait d'ailleurs comme un élément tout à fait central qui reboucle avec la question de la praxis (cf. supra). Si l'expérimentation peut se trouver indexée à d'autres valeurs qui ne sont pas progressistes comme la prise de risque, l'esprit d'entreprise et l'innovation (Spiro, 2012b ; Koh, 2014), elle se place néanmoins aussi au principe de nombre d'actions en ligne qui partent de structures diffuses dont le sens et la forme émergent précisément de l'action conjointe, mais ne la précèdent pas (Denouël, et al., 2014). Ces modes d'action typiques du web apparaissent à nombre de DHers comme des modèles à suivre, notamment en ce qu'ils se fonderaient sur un refus de l'idéologie (Scheinfeldt, 2012) au profit d'un faire s'inspirant d'un certain pragmatisme qui enjoint à passer d'abord par des expériences pratiques (les références à Dewey ne sont pas rares : Ramsay, Rockwell, 2012 ; Reber, Brossaud, 2007, etc.). Le politique promu par les humanités numériques relève donc plutôt de l'élaboration de valeurs ne précédant pas la capacité des individus à agir... via l'usage des TNIC. Pour Doueihi, le numérique serait ainsi « un système dynamique [...] habité par des identités polyphoniques capables de produire des comportements contestataires » (2013: 22). Outre le fait que, du côté DHers cet élan pragmatique de la dialectique moyen/fin peut aisément faire le lit d'un certain déterminisme technique (Hui Kyong Chun, Rhody, 2014), croire en un agir technologisé nécessairement créateur peut également, du côté des "prenants-part » conduire à d'évidentes méprises quant aux conditions de félicité de l'action en commun (Granjon, 2014b).

15 La valorisation du hack par les humanités numériques dessine donc un pragmatisme dont l'originalité est qu'il se fonde avant tout sur une matérialité technologique. Celui-ci est en effet largement orienté vers l'action et les conséquences pratiques que les outils qu'ils mobilisent permettent d'explorer. Comme nous l'avons déjà souligné, il rejette parfois l'idée d'un agir qui serait d'abord fondé en théorie ou en idéologie, au profit d'une expérimentation plus directe des programmes d'action des machines. Autrement dit, les valeurs ne précèdent pas les faits et surtout pas les scripts, c'est-à-dire les potentialités ouvertes par les appuis matériels. Les valeurs sont alors plutôt immanentes à l'agir technique: "C'est dans l'activité que se déterminent conjointement les fins et les moyens, qu'est établi ce qui est désirable et ce qui est digne d'intérêt » (Bidet et al., 2011 : 46). S'il y a production de "théorie", celle-ci est censée "surgir de l'action", de l'expérimentation d'hypothèses, notamment de celles portées par les outils numériques. Le pragmatisme technologisé des $\mathrm{HN}$ confie donc, dans ses penchants les plus technicistes, la force des analyses aux capacités (de calcul et de représentation) des technologies; technologies au nombre desquelles John Dewey comptait celles de l'esprit (l'idéal est pour lui une hypothèse dans une expérimentation, un outil pour l'action), mais que les DHers ont, pour certains d'entre eux, tendance à ne considérer que sous l'angle artefactuel des outils numériques.

16 Cet expérimentalisme pragmatique des HN s'appuyant sur des moyens techniques qui semblent générer leurs propres fins - et que leurs plus fervents zélateurs considèrent comme des formes pratiques de démocratie scientifique - nous semble porter en creux une vision politique où le succès de l'action se voit en grande partie confié à des scripts techniques, lesquels font de la question de l'accord un problème d'ajustement. Le commun produit de cette manière semble moins relever d'un travail collectif d'ajustement pratique à la participation d'autrui (la justesse des usages d'objets appareillant des valeurs) que d'une remise de soi et de son expérience à un dispositif qui régule le « vivre ensemble » plutôt qu'il ne permet de l'expérimenter (une délégation technologique de la 
justice). Si, comme le suggèrent Luc Boltanski et Laurent Thévenot, «l'engagement des objets oblige les êtres humains à être à la hauteur, à s'objectiver eux-mêmes en actionnant les objets, en les mettant en valeur » (1991: 166 - la fin justifie donc bien les moyens), encore faut-il ne pas oublier que les objets, en retour, mettent également en valeur les pratiques sociales et les personnes. Les moyens ont également des fins et c'est, là, leur manière de "participer ", de "prendre part " au cours de l'action. Leur justesse dépend moins de capacités intrinsèques à s'ajuster à la situation (les progrès de l'intelligence artificielle changeront peut-être la donne), qu'au fait de porter des programmes d'action en conformité avec les valeurs de ceux qui les mobilisent. Ce par quoi le collectif tient ne saurait ainsi se substituer intégralement à ce à quoi il tient (Bidet et al., 2011). Penser que les technologies puissent prendre en charge, sans reste, le système de valeurs de celles et ceux qui les mobilisent est assurément une illusion techniciste qui tend à faire de la technique un "procédé général " pouvant représenter à lui seul la dialectique (le continuum) moyens/fins. Si les moyens se trouvent justifiés par la fin, cette dernière a aussi besoin de justification, laquelle ne saurait être prise en charge par les moyens si ce n'est à considérer ceux-ci comme, finalement, la mesure de toute chose, par un amalgame du "mobile subjectif» et de «l'utilité objective». C'est précisément ce travers qui nous semble traverser, avec plus ou moins de force, certaines des implicites politiques liés au pragmatisme technologisé des humanités numériques.

Cette extension du domaine du faire vers le «technologique » est supposée permettre la création de "chaînes d'équivalences" constituées de fragments d'engagement et de subjectivités dont la mise au travail ne nécessiterait pas forcément d'accord préparatoire, si ce n'est quant à l'action à mener. De facto, ce pragmatisme technologisé permet, en certains cas, la conduite d'activités scientifiques et d'actions collectives particulièrement efficaces. Toutefois, valoriser l'expérimentation comme engagement dans un agir au détriment de l'expérience entendue comme "hypothèses théoriques " intégrant notamment des appuis normatifs n'est pas sans poser problème (pour Dewey, par exemple, la critique ne nécessite pas de fondement normatif et toute valuation est une critique). Le pragmatisme technologisé nous semble, en effet, parfois fleureter avec une vision postmoderne, apolitique et nébuleuse du pouvoir et des médiations. Sous couvert d'un détachement des préalables théoriques et idéologiques, lesquels sont censés faire obstacle plutôt que faciliter le déroulement des actions, on oublie que la technique est elle-même porteuse d'incitations, de cadres, d'obligations induisant un guidage des conduites qui n'est pas exempt de valeurs inscrites au cœur même du dispositif. Faire le pari de sujets sociaux rendus hautement compatibles par le partage de moyens techniques et leur fédération en groupes d'action scientifique ou politique, c'est postuler des identités professionnelles ou sociales mouvantes, instables, négociables, traversées de signifiants flottants et articulables par la technologie, ouvrant la possibilité de faire des expériences personnelles. Mais n'est-ce pas là une nouvelle version réductionniste de l' agir politique (au sens le plus large) confiné à des formes d'accrétion d'actions individuelles et instrumentées? Comme le relève Armand Mattelart pour les champ de l'action collective : «L'activité n'est pas une réponse, c'est une question » à laquelle il semble douteux de pouvoir répondre sans « [1]'inscrire dans un champ de détermination politico-idéologique » (2015), lequel ne saurait être ramené au seul statut d'hypothèses situées et révisables à tout moment. S'il est plaisant de considérer que la science ou le politique puissent se glisser dans l'ordinaire, l'imagination et les petites choses, il est fort risqué d'envisager, par exemple, que la lutte contre le pouvoir qui assujettit puisse être l'affaire de singularités individuelles, de micro-résistances autonomes et 
« encapacitantes », de subjectivités alternatives qui puissent ne jamais être en lien avec des sujets socialement solidaires et politiquement mobilisés par un projet collectif de réponse à l'ordre social.

Certains écrits des humanités numériques portent d'ailleurs d'autres exigences et conçoivent la critique comme une politique de la raison à partir de laquelle s'établissent des nécessités qui ne sont plus celles d'une science (fut-elle interdisciplinaire) supposément neutre, surplombante et dégagée des déterminations qui traversent la société dont elle est le produit, ni celles d'un pragmatisme technologisé, mais plutôt celles d'une pratique engagée de production de connaissances à visée synthétique (totalisation - cf. supra), indexée à l'exigence d'un travail du négatif axiologiquement fondé, mettant en cause les formes de domination, notamment issues de l'hégémonie capitaliste, et qui amoindrissent le sujet social. C'est à l'évidence le cas quand Jeffrey Schnapp, Peter Lunenfeld et Todd Presner (2009) affirment dans leur manifeste que «le droit d'auteur et la propriété intellectuelle sont des normes qui doivent être libérées de l'emprise du capital», assertion qui se trouve évidemment sous-tendue par une forte charge valorative. Un pan des HN est d'ailleurs parfois comparé à d'autres Studies critiques qui ont permis de considérer les formes de domination au-delà de la contradiction capital/travail, dans une perspective intersectionnaliste (Women, LGBTQ, Cultural, etc. - Kirschenbaum, 2012). Malgré cette parenté, certains autres de leurs territoires sont en revanche dénoncés pour leur manque d'attention aux rapports sociaux de race, de classe et de genre (Koh, Roopika, 2013 ; McPherson, 2012 ; Cong-Huyen, 2011).

Il est toutefois un domaine où les humanités numériques se penchent sur les formes de domination, c'est celui qui est lié à la sphère (de production) académique et à l'activité scientifique. Face à ces logiques d'exercice du pouvoir qui ne seraient que la traduction dans le champ des sciences des logiques dominantes traversant la société (Adema, 2012), les HN prétendent proposer une alternative aux modes de subjectivation et aux mises en disposition qui prévalent au sein du monde universitaire et ce, par la mise en œuvre de pratiques décentrant les habitudes du milieu. Elles proposent, en effet, de renouveler les règles et normes communes présidant au travail universitaire (écritures et publications collaboratives, remise en cause de l'auctorialité, construction de bases de données partagées, publicisation de carnets de recherche, curation, open peer review, open access, preprints, big et linked data, licences creative commons, open standards, etc. - Cavanagh, 2012), voire même de « hacker l'académie » (Cohen, Scheinfeldt, 2011 ; Fitzpatrick, 2011a), pour aller vers le développement d'une "science peer-to-peer " (McPherson, 2009; Thatcamp Paris, 2012), et pour certains, obliger aussi ladite science à assumer pleinement certaines responsabilités politiques: "À mon avis, ceux qui s'intéressent à "pirater l'académie" expriment trop peu d'intérêt à "pirater le monde", et les expérimentations académiques dans le domaine de l'open peer review ou des publications sous creative commons restent orientées autour des seuls intérêts universitaires " (Losh, 2012 : 176-177). Les HN se vivent notamment comme le lieu où les frontières entre la critique et la créativité deviennent arbitraires (Fitzpatrick, 2011b) et au sein duquel tomberaient les formes « d'antagonisme entre la subjectivation de soi comme chercheur que valorise le champ académique et la subjectivation de soi comme auteur créatif » (de Lagasnerie, 2011 : 70). Elles revendiquent ainsi de nouvelles valeurs à inscrire : a) dans les institutions scientifiques qui continuent notamment à prendre part à " une économie politique fondée sur les intérêts hérités de l'imprimé » (Adema, 2012); et b) dans les corps, de nouvelles règles pour la pratique et un nouvel ethos pour le chercheur. Dans cette 
perspective, les humanités numériques sont envisagées comme montrant le chemin dans la mesure où elles donneraient d'ores et déjà « lieu à de nouvelles formes de la recherche académique, de nouveaux moyens d'évaluer et d'organiser la connaissance humaniste, et de nouvelles formes de communication culturelle »(Svensson, 2010).

\title{
Faire science autrement : débats autour de la production et de la propriété scientifique
}

\author{
« Nous voulons en effet créer des connexions \\ nouvelles entre des totalités bien articulées et non \\ juste quelques vagues dans une mer sans \\ mouvement. " \\ Paul Feyerabend, Contre la méthode. Esquisse d'une \\ théorie anarchiste de la connaissance.
}

Les humanités numériques s'interrogent donc sur le rôle du savoir scientifique qu'elles produisent. Elles estiment devoir ouvrir la production scientifique à des non-spécialistes : à ses entours d'abord, à des personnes hautement qualifiées qui ne disposent pas nécessairement de postes dans le champ académique, mais plus largement aussi à des "profanes" qui souhaiteraient collaborer sans pour autant devoir se prévaloir de compétences scientifiques particulières. Elles remettent donc en cause, ce que Marx, en son temps, décrivait comme la division entre le travail matériel et le travail intellectuel, entre des spécialistes de la théorie et des profanes qui n'habiteraient que la pratique. S'il s'agit, pour le social scientist œuvrant au sein des HN, de ne pas «nourrir le fantasme [que sa conscience] est indépendante de la réalité matérielle » (Eagleton, $2000: 16$ ), il lui faut également considérer que la production du savoir scientifique au sein des SHS peut être diversement ouverte à des non spécialistes, et ce, notamment grâce au numérique (Doueihi parle d'ailleurs à cet égard de «numériciens par accident»-2008). Cette revendication, portée par « certaines catégories socioprofessionnelles traditionnellement dominées dans les milieux universitaires " (Citton, 2015: xx), prend notamment forme dans le mouvement états-unien des alternative academics («alt-ac jobs»), lesquels « promeuvent les métiers nouveaux exercés par des techniciens lettrés, ou des lettrés techniciens, qui ne sont pas intégrés à l'université comme chercheurs ni comme membres d'une unité de service " (Berra, 2012; Flanders, 2012), ou bien par des scientists en position notoire de précariat (Hui Kyong Chun, 2013; Grusin, 2013). Les HN souhaitent donc en quelque sorte «démocratiser» l'activité scientifique en valorisant la participation et la collaboration (crowdsourving, co-curation, etc.), mais également en ouvrant la réception, en partageant davantage, en explorant des formes alternatives de communication savante utilisant des technologies et des canaux grand-public (QR Code, UGC, blogs, Twitter, etc. - Adema, 2012 ; Carter, 2013 ; Warwick et al., 2012 ; Boismenu, Beaudry, 2007) et donc en travaillant à ne pas réserver la production scientifique à quelques cénacles de lecteurs qui seraient essentiellement constitués de pairs : « S'il est une raison qui empêche de faire laboratoire comme au bon vieux temps des sciences de la nature, c'est que tous ces outils utilisés par les SHS notamment sont entre les mains de tous et qu'ils gagnent, grâce au réseau, une puissance de diffusion incontrôlable " (Boullier, 2007: 18). Les HN permettraient ainsi que se développent de nouvelles interactions «entre la recherche scientifique et technique, les citoyens et les organisations de la société civile » (Aigrin, 2011: 84 ; Dacos, 2010), en mettant notamment 
en avant la figure du «lecteur inattendu " (unexpected reader), extérieur au monde universitaire mais capable, par un accès facilité aux ressources scientifiques, d'aider à des avancées scientifiques ${ }^{5}$ (Smith, 2011). Sans aller jusqu'à voir là, bien imprudemment, une remise en cause radicale des « asymétries de savoirs et de pouvoirs » (Boullier, $2007: 18$ ), il apparait cependant évident que les potentialités de partage ouvertes par les TNIC à destination du plus grand nombre permettent de déstabiliser « le souci aristocratique de maintenir le réel à distance, [qui] continue de frapper les spécialistes de la pensée [...] peu soucieux de toute façon d'adresser leurs "papiers" à un autre public que celui que constituent leurs collègues" (Dericquebourg et al., 2012: 12). Donner accès ne revient toutefois jamais à s'assurer d'intéresser, et la seule mise en visibilité ne peut être synonyme de mise en publicité (débats, appropriations). La critique invite à ne pas penser seulement l'aval de la production théorique, mais aussi son amont, en se plaçant à l'endroit du sujet pratiquement engagé, avec des armes complémentaires aux siennes, afin notamment de l'encourager dans ses efforts de distanciation ${ }^{6}$. Mais encore faut-il se garder de projeter, sur lui, certaines des formes idéalisées du débat intellectuel, c'est-àdire sans jamais perdre de vue ce que sont les conditions réelles d'accès à la totalité, à l'universalisme abstrait et à la production scientifique/politique dont fait très vite fi l'idéologie de la participation par le numérique.

21 Portées par des manières de faire science plus « collaboratives et génératives » (Burdick et al., 2012 : 3), les humanités numériques s'inscrivent notamment au sein du débat juridique qui a été lancé, au tournant des années 2000, sur les questions de propriété intellectuelle (Doueihi, 2008), lesquelles se trouvent au cœur de la puissance consacrée des industries de l'édition universitaire. Ces dernières ont fait reconnaître et appliquer, par des actions juridiques ciblées, les principes des droits d'auteur et d'éditeur, ce qui leur a permis de contrôler la reproduction des « œuvres de l'esprit » et de s'assurer des rentes de situation en cas de succès, mais aussi de ne pas rémunérer l'essentiel des forces productrices en cas d'échec (Hesmondhalgh, 2007). L'application du droit d'auteur à la production scientifique a également permis d'entretenir une certaine "aura» des productions scientifiques et de charrier un imaginaire consacrant le mythe du chercheur (issus de celui de l'artiste). Les DHers dénoncent la prégnance de ce mythe dans l'imaginaire associé à la recherche scientifique où le "génie créateur » œuvrerait seul à un travail d'ordre symbolique. Face à l'idée d'une science réservée dont les travaux et les résultats devraient être de plus en plus protégés et brevetés au nom du respect de l'auctorialité, ils opposent l'idée d'une science qui pourrait s'avérer nettement plus ouverte et collaborative en se détachant du principe de paternité des œuvres (authorship) :

« Nous n'imaginons plus la création intellectuelle comme un travail autonome ou comme le résultat du travail d'un génie solitaire (image dont il est certain que la réflexion critique a cherché à déconstruire depuis des décennies). Nous pensons plutôt la captation et l'expression des énergies de création d'une communauté toujours croissante et virtuellement illimitée de praticiens. [...] Nous passons d'une ère où l'élaboration théorique reposait sur l'auteur individuel du "grand livre" à une ère où elle s'appuie sur les possibilités de la réalisation collaborative liée au "grand projet" " (Burdick et al., 2012: 83).

$\mathrm{Au}$ sein des $\mathrm{HN}$, la prédominance de l'utilisation de l'information automatisée et l'augmentation conséquente des tailles des projets fait en effet de « la collaboration [...] la règle plutôt que l'exception» (Hayles, 2012: 51). John Unsworth évoque à cet égard un "déplacement d'un modèle coopératif vers un modèle collaboratif: dans le modèle coopératif, l'individu produit du savoir qui se réfère et puise dans le travail d'autres 
individus; dans le modèle collaboratif chacun travaille en conjonction avec les autres, produisant conjointement du savoir qui ne peut être attribué à un seul auteur » $(2003: 6)$. Ces principes de collaboration valorisés dans la mesure où ils peuvent apporter «beaucoup de bénéfices intellectuels, professionnels et sociaux» (Pitti, 2004: 485) ne devraient, ainsi, plus être contraints dans leurs développements par les habitudes induites par les modèles (de droit) d'auteur. En rapport avec les revendications mentionnées supra sur la nécessité d'ouvrir le champ de la recherche académique, les humanités numériques se proposent donc aussi de penser autrement la paternité des travaux de recherche ayant nécessité le concours, à des étapes diverses, d'un ensemble d'acteurs. Nombre d'articles s'affairent ainsi souvent à la présentation détaillée de projets de production, de partage en ligne des savoirs ou de projets collaboratifs ayant connu des résultats intéressants et pour lesquels est alors analysé le potentiel d'une approche collaborative. Katherine Hayles (2012) insiste par exemple sur l'intérêt d'un ensemble de projets (Virtual Plowshares, Hypermedia Berlin, Alt-X Online Network, etc.) en ce qu'ils permettent «à des étudiants, même en premier cycle universitaire, de faire des contributions significatives", ce qui, souligne-t-on, ne leur aurait pas été permis dans une vision de la production de savoir s'appuyant sur un imaginaire lié à la valorisation personnelle de l'auteur universitaire. Ces premières positions sont un point de départ intéressant. Elles posent les contradictions entre les nouvelles formes du travail universitaire et la régulation de ses outputs, mais ne sont-elles pas quelque peu timides dans leur remise en cause des questions de propriété intellectuelle et de copyright? Si l'on peut, par exemple, apprécier la manière qu'a Doueihi de lier la question des nouvelles pratiques collaboratives dans la recherche à celles émergeant dans d'autres secteurs posant ainsi les jalons d'une critique générale des limites des droits de propriété intellectuelle -, on peut néanmoins regretter que ces enjeux de régulation, ne l'enjoignent guère à développer une critique plus serrée que celle qui consiste à observer que «la technique et les pratiques qu'elle autorise sont en train de nous pousser à repenser et à reformuler non pas seulement les lois concernant le copyright et la propriété intellectuelle mais surtout la notion même d'auteur " (Doueihi, 2011: 118). Le plus souvent, la plupart des DHers semblent vouloir en rester à une vision technodéterministe dans laquelle l'utilisation des techniques viendrait " naturellement » bouleverser les équilibres que pose la régulation (Wharton, 2013). Aussi, est-il bien difficile, malgré les arbitrages récurrents des HN en faveur du «faire» et du hack (cf. supra), de trouver des invites à "prendre les choses en main» du point de vue de la propriété intellectuelle, si ce n'est, parfois, dans le but de mieux savoir... comment s'y conformer?.

23 C'est sans doute sur le thème de l'open access (Vecam, 2011; Dacos, Mounier, 2010 ; Dacos, 2010 ; Magis, Granjon dans ce numéro) que la discussion semble la plus avancée. Depuis l'appel de la Budapest Open Archive Initiative (2002) et la Déclaration de Berlin sur le libre accès à la connaissance ${ }^{8}$ (2003), consacrant l'idée que « la recherche sur fonds publics doit revenir au public, à la fois pour des raisons éthiques, sociétales et scientifiques " (Dacos, 2012a : 218), le principe du libre accès repose la question de la place et de la fonction sociales de la production théorique. Dans une perspective proche à certains égards de certaines thèses du capitalisme cognitif (Moulier-Boutang, 2007) et appuyant leurs argumentations sur les particularités économiques des biens numériques - " non excluabilité et non rivalité, reproduction illimitée pour un coût marginal proche de zéro " (Peugeot, 2011: 19; cf. infra) -, certains DHers condamnent la privatisation et la 
marchandisation de la connaissance, notamment à l'heure numérique (Forum Social Mondial, 2009) et militent pour un principe d'ouverture et de partage larges de l'information scientifique. Face à la mainmise d'une poignée d'éditeurs puissants et vendant à prix d'or, aux institutions de recherche, les abonnements à des revues permettant à leurs membres d'accéder notamment à leurs propres articles, certaines propositions appuient l'importance du partage en ligne des textes scientifiques par des personnes qui collectivement trient, classent, référencent, commentent et mettent à disposition des œuvres avec une qualité comparable, voire supérieure, à celle de certain catalogues payants (Faucilhon, 2010). Les propositions autour du libre accès portent ainsi l'idéal futur d'un monde de la recherche ouvert et réconcilié avec le monde nonacadémique auquel il rendrait les fruits de la production scientifique en tant qu'ils sont considérés comme des communs de la connaissance (Hess, Ostrom, 2007; Vecam, 2005 ; 2011).

Les problématiques liées au libre accès permettent ainsi, aux HN, de poser en pratique la question des communs de la connaissance, mais c'est pour généralement en tempérer les potentialités les plus critiques en recourant à une logique de l'adaptation à l'économie dominante. Alors même que beaucoup des travaux relevant des humanités numériques appuient la "puissance du nombre " (Dacos, 2012a) et les effets de réseaux importants que le numérique permet d'engendrer, il est dommage qu'une question comme celle du financement, de la diffusion et de l'ouverture des productions scientifiques ne soit pas posée politiquement plus avant au sein d'un collectif critique rassemblant enseignantschercheurs et lecteurs, bien au-delà des communautés étroites habituellement considérées. Au lieu de cela, certains DHers semblent privilégier la voie opposée ${ }^{9}$ et importer, dès lors, l'idée prégnante de bon nombre de débats sur les communs de la connaissance selon laquelle la gestion de ceux-ci est pensée comme une sorte de one best way possible mais à choisir au cas par cas, «[a]u regard de la taille de la communauté concernée ou de la lourdeur des investissements nécessaires pour faire grandir cette ressource » (Peugeot, 2012 : 22). En conséquence, qu'elles soient ou non ancrées dans une problématique des communs, si les HN portent une possible critique de la propriété intellectuelle, elles sont pour autant encore loin de s'attaquer véritablement et politiquement à la propriété elle-même - et encore moins aux mutations des modèles d'exploitation du travail centré, en régime capitaliste, sur cette catégorie. Et si Jeffrey Schnapp, Peter Lunenfeld et Todd Presner affirment dans leur manifeste que «le copyright et les standards de propriété intellectuelle doivent être libérés de l'emprise du Capital, y compris le capital appartenant aux héritiers ayant-droit qui vivent en parasites sur les réalisations de leurs défunts prédécesseurs", les propositions d'«action de guérilla » auxquelles ils invitent afin de mettre en œuvre cette critique (en utilisant de manière illégale des ressources " copyrightées ») semblent somme toute assez dérisoires au regard d'un tel projet. Elles se rabattent, de surcroît, sur la défense d'une forme de propriété intellectuelle, certes probablement moins expropriable que ce qu'offre la particularité du système américain de copyright (qui ne reconnaît pas de « droit moral » à la différence des droits d'auteurs continentaux), mais tout de même liée à la personnalité créatrice des «makers». Ce type de proposition ne remet pas franchement en cause le mode de production capitaliste. Tout au plus en souligne-t-il la nécessité d'en adapter certains éléments, dans l'optique d'améliorer son efficacité globale, par exemple en limitant la transmissibilité des droits de l'auteur vers une institution ou vers un descendant, ou en favorisant les modèles hybrides d'appropriation public/privé (quand bien même ces «partenariats » peuvent être par ailleurs critiqués - Renaud, 2005). Dès 
lors, on peut se demander jusqu'à quel point ces propositions ne sont pas déjà réintégrées (ou en voie de réintégration) par les mutations du capitalisme, comme le remarque Isabelle Garo dans une critique des discours issus de la " culture numérique » :

«Bien entendu, telle n'est pas l'optique des nouveaux militants des communs, qui soulignent avant tout le caractère anticapitaliste de ce type de bien et le rattachement à l'idée d'un patrimoine commun de l'humanité, qui ne saurait être marchandisé. Mais de l'exception culturelle juridiquement protégée aux biens communs rénovés, la perturbation toute relative de la liberté des marchés n'entamera en rien un fonctionnement économique et social d'ensemble, capable d'une grande souplesse, apte à retourner ses contradictions en atouts et parfois même ses contradicteurs en alliés. » (Garo, 2013 : 247).

D'ailleurs, en n'investissant pas vraiment la question de la propriété intellectuelle du point de vue d'une critique politique posant la question de la propriété dans le système capitaliste, les positions des digital humanities développent parfois des préoccupations relativement proches de celles qui émergent dans les "industries créatives", et par lesquelles elles sont souvent déjà dépassées. Ainsi, Nicolas Colin, co-fondateur de TheFamily et co-auteur avec Henri Verdier d'un ouvrage intitulé L'âge de la multitude et sous-titré Entreprendre et gouverner après la révolution numérique (2012), n'hésite pas à s'appuyer sur les thèses d'Antonio Negri et Michael Hardt (2004) et celles des théoriciens du capitalisme cognitif pour défendre la vision d'un «monde hyperfluide » au sein duquel l'économie se base sur les "flux d'innovations, d'information et de connaissances", lesquels seraient aujourd'hui le centre de la valorisation. Ici, comme pour les thuriféraires du libre accès, "les tendances immanentes au capitalisme contemporain sont censées participer à la formation sous nos yeux d'un autre monde, donnant la place centrale à la connaissance, à la créativité et à l'autonomie censées les accompagner comme leur ombre» (Garo, 2013: 232). Les HN les plus critiques, qui ont réfléchi la mystification induite par ces métaphores faisant du cyberespace un ailleurs perdu dans l'éther ont pourtant insisté sur le fait que "les ordinateurs créent l'illusion de l'immatérialité. Il incombe [alors] aux humanistes numériques, non seulement de révéler cette illusion pour ce qu'elle est, de révéler le caractère matériel [thingness] du numérique, mais aussi d'interpréter les effets de l'illusion dans un contexte culturel » (Jones, 2014 : 131). De facto, les supposés mondes numériques, comme les subjectivités amenées à les arpenter ou s'en servir nécessitent des ancrages multiples dans le monde physique : écrans, interfaces d'accès, disques de stockage, émetteurs et récepteurs de signaux radio/téléphonique/ GPS, etc., auxquels il faudrait aussi ajouter d'autres processus, tout aussi matériels comme le travail des techniciens « dont la dépense d'énergie et le stress sont tout ce qu'il y a de plus matériel en étant pourtant occasionnés par une tâche essentiellement intellectuelle » (Harribey, 2004 : 163). Certaines HN semblent donc armées pour critiquer la vision d'une économie en apesanteur qui disqualifierait toute approche matérialiste sous prétexte que l'essentiel de l'économie et de ses catégories serait « immatériel». Elles semblent également équipées pour critiquer l'essentialisation des principes économiques en fonction de particularités ontologiques que les objets que l'économie (de la connaissance) administre contiendraient en propre : «renoncer [...] à l'idée qu'il existe des choses par nature inappropriables pour fonder vraiment et entièrement en droit l'inappropriabilité » (Dardot, Laval, 2014: 37). Pour autant, ces problématiques restent par trop éparses et ne se constituent pas, pour l'heure, sur ce terrain, ce qui limite leur portée. Par conséquent, si l'on peut considérer que les HN ouvrent des brèches par lesquelles se créent de nouvelles manières de faire préparant un possible changement radical, il conviendrait toutefois d'en exacerber la dimension politique : aller vers un au- 
delà de l'économie de l'édition scientifique en régime numérique conduisant à un au-delà de l'économie capitaliste.

De surcroît, le modèle original des communs mis en avant par Elinor Ostrom appuie l'idée que la meilleure gestion d'un type de bien particulier, non-exclusif mais rival ${ }^{10}$ (les communs naturels ou «common-pool ressources" - e.g. les zones de pêche) pouvait s'organiser hors de l'alternative entre le marché privé et la propriété publique, via certains modèles de gestion collective portés par des communautés (Ostrom, 1990). Son adaptation à la question de la connaissance, a fortiori à l'ère numérique, enjoint de réfléchir aux spécificités des communautés organisées autour de ces biens et qui entendent en exacerber les caractères les plus incompatibles avec un modèle d'appropriation privé. Au fondement des propositions demeure donc une forme d'essentialisation des particularités des biens considérés - que Pierre Dardot et Christian Laval appellent «réification du commun» (2014: 32). Ce seraient ainsi des caractéristiques « naturelles » de la connaissance (difficile exclusion, faible rivalité, etc.) qui en feraient des biens communs et en légitimeraient une gestion plus collective, laquelle n'est que rarement pensée comme pouvant participer d'une dynamique plus large susceptible de dépasser le domaine de pratiques considéré, c'est-à-dire sans remettre en question « la rationalité des marchés ou de l'État » (Dardot, Laval, 2014 : 155). Dès lors, au-delà de l'attention fine - et légitime pour n'en pas réduire l'importance portée par les HN aux changements induits par les possibilités techniques et ce que les nouveaux usages qui se développent autour de ces possibilités contiennent de changement radical potentiel, on peut se demander jusqu'à quel point leurs propositions sont une invite à une prise en main politique de la question. Si, "[a]ujourd'hui, le numérique offre encore plus de possibilités pour empêcher la main-mise sur les œuvres, pour faire circuler par-delà les barrières le savoir et l'émotion » (Le Crosnier, 2005 : 222), les défenseurs les plus avisés des communs ne sont pour autant pas dupes du fait que « les nouvelles capacités sont immatures, fragiles, susceptibles d'être perverties ou détournées de leur potentiel» (Aigrin, 2005: 32). Or dans bon nombre de travaux relevant des humanités numériques, on constate une foi peu prudente en ce que la connaissance en tant que commun, associée à la numérisation de ses outputs, contiendrait en propre, quasiontologiquement, une limitation de son appropriation capitalistique (Peugeot, 2011 ; Kill, 2012). Ici encore, la revendication semble, la plupart du temps, vouloir se limiter à défendre la préservation d'« un juste équilibre entre domaine privé et domaine public, sans éroder le domaine public » (Li, $2011: 257)$ et d'une reconnaissance institutionnelle de la pluralité des pratiques d'appropriation pour «laisser ouverts les chemins de la découverte " (Hess, Ostrom, 2007: 8). Mais cette conviction risque d'être rapidement ébranlée si la critique potentielle portée par les communs de la connaissance dans le monde numérique n'est pas implémentée au sein de la pratique sociale. Ceci nécessite de construire la question de la propriété en une véritable question politique globale portant à un plus haut degré de nécessité la contradiction "entre un savoir qui ne vaut socialement que par sa diffusion large, mais qui [concrètement] ne se valorise économiquement que par les limites imposées à son accès » (Garo, $2013: 247)$.

\section{$* * *$}

Discipline, multidiscipline, transdiscipline, nouveau paradigme, révolution médiatique (Schnapp et al., 2008), «nouvelles formes de création et de dissémination des savoirs » 
renouvelant «matériaux, méthodes et [herméneutiques] (Dhiparis, 2013), extension du domaine des SHS ne se résumant pas à «la simple introduction des technologies numériques dans le processus de recherche, ni même comme l'informatisation de celleci » (Dacos, 2012b), nouveau rapport à soi et à la société, développement d'une « recherche connectée, collaborative, horizontale, multimodale, multidisciplinaire et multilingue » (Dhiparis, 2013), « chapiteau » rassemblant « un large spectre d'approches académiques, vaguement reliées entre elles par un intérêt commun en matière de technologie et de la recherche humaniste » (Terras, 2012) : les définitions et attendus des humanités numériques sont pour le moins variés. Ils les font se tenir quelque part entre " entre mission de service et révolution épistémologique " (Berra, 2012) et ne décrivent en rien un champ unifié dans la mesure où territoires et frontières sont encore en définition ${ }^{11}$ (Svensson, 2010 ; Ramsay, 2011). De facto, le rapport des humanités et des SHS au numérique revient à une mise en mouvement des premières par le second : nouveaux observables, nouvelles techniques d'enquête et d'administration de la preuve, nouveaux processus de traitement des données empiriques, de collaboration, d'écriture, de diffusion, d'évaluation, etc. (Fyfe, 2012). Le numérique, élément qui, du fait d'une présence accrue de dispositifs sociotechniques et tout particulièrement de l'informatique connectée, se retrouve au sein de la quasi-totalité des sphères sociales des sociétés capitalistes avancées et constitue actuellement l'un de nos milieux associés les plus prégnants (Simondon, 2012), vient aussi modifier certaines manières de faire et de penser des disciplines scientifiques (Schreibman et al., 2004). Ce déplacement est-il pour autant un mouvement critique? Assurément, la réponse est positive quand les humanités numériques mettent en exergue la domination technoculturelle (Schnapp et al., 2008), s'intéressent aux logiques des nouvelles structures productives des «industries créatives ", au guidage des conduites par le code, à la mise à mal de l'« éthique hacker » par la logique marchande (Himanen, 2001 ; Oliver et al., 2011) et qu'elles font comprendre que les technologies sont des formes d'objectivation de valeurs sociales inscrites dans des scripts (des programmes d'action, des codes techniques) qui participent pleinement à l'établissement d'une certaine hégémonie sociale. Les HN sont également critiques quand elles s'appuient sur l'enchevêtrement d'intérêts de connaissance et d'émancipation qui, à l'occasion du digital turn, conduisent à réintroduire des problématiques autour des rapports sociaux de production, de la propriété lucrative, de la forme marchandise, de la valeur d'usage, du travail abstrait, ou encore du piratage de la valeur opéré par la capitalisme (Aigrin, 2005 ; Lessig, 2008 ; Bollier, 2014) ; autant de thématiques qui mettent au cœur de leurs réflexions les conventions capitalistes prévalant au sein des sociétés contemporaines, ainsi que l'évolution de ces dernières sous le coup de la numérisation du signe :

« De fait, il y a souvent une dimension politique ou militante à l'engagement dans les humanités numériques : au sein des communautés savantes, pour changer leurs méthodes, comme au sein de la société en général. En ce sens, on peut dire qu'il s'agit d'un mouvement, et non plus seulement d'un moment. Ce mouvement a produit ses manifestes. Ils font entendre la voix d'une minorité et expriment un sentiment d'oppression - et probablement, dans le même temps, le sentiment de distinction d'une avant-garde clairvoyante » (Berra, 2012).

Mais la perspective critique n'est pas pour autant la chose la mieux partagée au sein des humanités numériques. D'aucuns suggèrent même que la critique (empruntant pour l'occasion la « figure de l'intellectuel » et le legs sartrien comme repoussoirs) ne pourrait faire que mauvais ménage avec le numérique ou que les SHS auraient à devenir 
« postclassiques » pour s'y ajuster (Wieviorka, 2013: 34 et suiv.). Michel Wieviorka va, là, un peu vite et fait notamment abstraction, au-delà des recherches menées par quelques collègues fréquentant la même institution que lui (plus ils sont proches, plus leurs recherches sont évidemment positivement qualifiées ${ }^{12}$ ), des travaux foisonnants en SHS portant sur les enjeux et usages des technologies numériques, lesquels furent développés avant même l'émergence de l'Internet grand-public et dont, en France, la revue Réseaux est l'un des grands témoins (cf. le n 184-185 sur les 30 ans de la revue ; Denouël, Granjon, 2011). Quant à une critique qui, nous dit-on, ne serait capable que de "réciter une nouvelle version de la sociologie critique vantant les merveilles de l'autoproduction populaire et des résistances à la domination » (Boullier, $2007: 20$ ) et serait dépourvue des qualités nécessaires pour dialoguer avec les humanités numériques, nous pensons avoir précisément démontré le contraire. D’une part, parce que la critique (que nous ne saurions confondre avec l'activité des think tanks du blairisme - Wieviorka, 2013:59) a pour tradition de s'intéresser aux évolutions technoculturelles des sociétés capitalistes avancées (pensons par exemple aux travaux d'Andrew Feenberg - 2014 -, ou à ceux de Christian Fuchs - 2013) et, d'autre part, parce qu'une partie des HN envisage aussi le numérique comme un répertoire de potentialités permettant d'appareiller (au triple sens d'outiller, de coupler et de démarrer) des conduites de dévoilement, de résistance et d'émancipation, allant directement à l'encontre de certaines formes de domination.

Bien évidemment, répétons-le, cette pente n'est pas nécessairement la mieux partagée au sein du mouvement et Alan Liu n'a pas manqué de souligner que :

«Rares sont [les DHers] qui étendent leur critique au registre complet de la société, à l'économie, à la politique ou à la culture. La question de savoir si, aujourd'hui, les humanités numériques participent ou résistent aux grands flux postindustriels, néolibéraux, entrepreneuriaux et mondiaux du capitalisme informationnel est une question rarement entendue au sein des associations scientifiques, des conférences, des revues et des projets avec lesquels je suis familier. Pas même sous les formes les plus stéréotypées - par exemple "la fracture numérique", la "surveillance", la "vie privée", les “droit d'auteur" -, ces questions ne sont pas posées » (Liu, 2012 : 491).

De surcroît, la critique s'avère parfois parasitée par un penchant techniciste qui aurait tendance à accorder un primat aux structures technologiques et ferait du digital turn la source de tout changement, plutôt que l'une des médiations (fut-elle centrale), par le biais desquelles s'exercent des logiques de domination qui ne sont «techniques » que dans leurs moyens (assignés à des fins notamment marchandes) et ne constituent donc pas un ordre autonome (Bianco, 2012; McPherson, 2012). À l'instar de Jacques Ellul (2008) qui voyait dans la technique le facteur décisif des sociétés contemporaines, les HN les plus critiques remplacent par exemple facilement la centralité du capital par celle du numérique, envisagé alors comme " procédé général » portant une fin et configurant des rapports sociaux (quant à celles qui ne le sont pas, elles tendent le plus souvent à tomber dans un utopisme qui fait de la technologie la nouvelle panacée - Hui Kyong Chun, 2013). Mais a contrario, il serait tout aussi contreproductif de considérer la numérisation du signe comme n'affectant pas, à la fois la base matérielle, les superstructures, et les existences qui participent des formations sociales qui l'ont largement adoptée. Si les fins se donnent des moyens, ceux-ci sont également porteurs de fins et c'est là une de leurs caractéristiques centrales (Latour, 2000). La critique se doit d'intégrer le domaine technique à son champ d'exercice et assurément, les humanités numériques peuvent l'y aider. De leur côté, ces dernières doivent s'efforcer de ne pas considérer la critique comme une breloque poussiéreuse de la brocante académique au prétexte que le monde 
numérique, immatériel et connecté, serait déjà, d'une certaine manière, post-critique (ce qu'elles laissent entendre de manière assez claire lorsque certains auteurs affirment qu'il est désormais temps de «critiquer le mantra de la critique " - Davidson, Goldberg, 2004 : 65). Face au risque d'une telle mystification, il convient de rappeler que la catégorie centrale de matérialité des analyses marxiennes ne conduit pas Marx à négliger les activités intellectuelles et, par ailleurs, que la critique matérialiste s'avère indispensable pour conduire une analyse conséquente d'une autre catégorie largement répandue au sein des $\mathrm{HN}$ : celle de l'immatériel.

\section{BIBLIOGRAPHIE}

Adema (Janneke), « Mettre en pratique ce que l'on prêche. La recherche en sciences humaines et sa praxis critique ", in Mounier (Pierre) dir., Read/Write Book 2. Une introduction aux humanités numériques, Marseille, OpenEdition Press, 2012, http://books.openedition.org/oep/254.

Aigrin (Philippe), Cause commune. L'information entre bien commun et propriété, Paris, Fayard, 2005.

Aigrin (Philippe), « De l'accès libre à la science ouverte ", in Vecam (ed.), Libres savoirs. Les biens communs de la connaissance, Caen, C\&F, 2011, p. 77-85.

Bachimont (Bruno), « Le nominalisme et la culture : questions posées par les enjeux du numérique », in Stiegler (Bernard) dir., Digital Studies. Organologie des savoirs et technologies de la connaissance, Paris, IRI/FYP, 2014, pp. 63-78.

Berlan (Aurélien), La fabrique des derniers hommes. Retour sur le présent avec Tönnies, Simmel et Weber, Paris, La Découverte, 2012.

Berra (Aurélien), « Faire des humanités numériques », in Mounier (Pierre) dir., Read/Write Book 2. Une introduction aux humanités numériques, Marseille, OpenEdition Press, 2012, http:// books.openedition.org/oep/238.

Berry (David M.), Critical Theory and the Digital, New York, Bloomsbury, 2015.

Bianco (Jamie), « This Digital Humanities Wich Is Not One », in Gold (Matthew K.) ed., Debates in the Digital Humanities, Minneapolis, University of Minnesota Press, 2012, pp. 96-112.

Bidet (Alexandra), Quéré (Louis), Truc (Gérôme), « Ce à quoi nous tenons. Dewey et la formation des valeurs ", in Dewey (John), La formation des valeurs, Paris, La Découverte, 2011, pp. 5-64.

Boismenu (Gérard), Beaudry (Guylaine), « Du concept à l'outil : penser et instrumenter l'édition numérique de revues savantes ", in Brossaud (Claire), Reber (Bernard), Humanités numériques 2. Socio-informatique et démocratie cognitive, Paris, Hermes/Lavoisier, 2007, pp. 181-194.

Bollier (David), La renaissance des communs, Paris, Charles Léopold Mayer, 2014.

Boltanski (Luc), Chiapello (Ève), Le nouvel esprit du capitalisme, Paris, Gallimard, 1999.

Boltanski (Luc), Thévenot (Laurent), De la justification. Les économies de la grandeur, Paris, Gallimard, 1991. 
Boyd (Danah), Crawford (Kate), « Six Provocations for Big Data », A Decade in Internet Time: Symposium on the Dynamics of the Internet and Society, 2011, http://ssrn.com/abstract=1926431

Brohm (Jean-Marie), Les principes de la dialectique, Paris, Les Éditions de la passion, 2003.

Brohm (Jean-Marie), « Louis Althusser et la dialectique matérialiste », in Vincent (Jean-Marie) dir., Contre Althusser, Paris, UGE, 1974, pp. 15-92.

Boullier (Dominique), « Préface. La nouvelle fabrique des SHS », in Brossaud (Claire), Reber (Bernard), Humanités numériques 1. Nouvelles technologies cognitives et épistémologies, Paris, Hermes/ Lavoisier, 2007, pp. 17-21.

Burdick (Anne), Drucker (Johanna), Lunenfeld (Peter), Presner (Todd), Schnapp (Jeffrey), Digital Humanities, Cambridge MA, MIT Press, 2012.

Burnard (Lou), « Du literary and linguistic computing aux digital humanities : retour sur 40 ans de relations entre sciences humaines et informatique ", in Mounier (Pierre) dir., Read/Write Book 2. Une introduction aux humanités numériques, Marseille, OpenEdition Press, 2012, pp. 45-58.

Carter (Bryan W.), Digital Humanities. Current perspective, Practices and Research, Howard House, Emerald, 2013.

Casilli (Antonio), Tubaro (Paola), « Why Net Censorship in Times od Political Unrest results in More Violent Uprisings: A Social Simulation Experiment on the UK Riots », Bulletin of Sociological Methodology, vol. 115, $\mathrm{n}^{\circ}$ 1, 2012, http://hal.archives-ouvertes.fr/docs/00/68/23/17/PDF/ CasilliTubaro_ICCU_HAL.pdf.

Cavanagh (Sheila), « Living in a Digital World : Rethinking Peer Review, Collaboration and Open Access ", Journal of Digital Humanities, vol. 1, n 4, 2012, http://

journalofdigitalhumanities.org/1-4/living-in-a-digital-world-by-sheila-cavanagh/.

Cecire (Natalia), « Introduction: Theory and the Virtues of Digital Humanities », vol. 1, n 1 , Journal of Digital Humanities, 2011, http://journalofdigitalhumanities.org/1-1/introductiontheory-and-the-virtues-of-digital-humanities-by-natalia-cecire/.

Christ (Julia), « La culture - un partenaire d'interaction? Questions de méthode pour une théorie critique ", in Noppen (Pierre-François), Raulet (Gérard), Macdonald (Iain) dir., les Normes et le possible. Héritage et perspectives de l'École de Francfort, Paris, Éditions de la maison des sciences de l'homme, 2012, pp. 71-89.

Cohen (Dan), Scheinfeldt (Tom) eds., Hacking The Academy. A Book Crowdsourced in One Week, Ann Arbor, Digital Culture Book/University of Michigan, 2011, http://www.samplereality.com/wpcontent/uploads/2011/09/Hacking_the_Academy.pdf.

Collectif, THATCAMP PARIS 2012. Non-actes de la non-conférence des humanités numériques, Paris, Éditions de la Maison des sciences de l'homme, 2012, http://books.openedition.org/ editionsmsh/278.

Cong-Huyen (Anne), « Toward an “Asian American” Digital Humanities », 2011, http:// anitaconchita.wordpress.com/2011/01/10/an-asian-american-digital-humanities-or-digitalasian-american-criticism/.

Dacos (Marin), «Vers des médias numériques en sciences humaines et sociales : une contribution à l'épanouissement de la place des sciences humaines et sociales dans les sociétés contemporaines ", Tracés, $\mathrm{n}^{\circ}$ hors-série, 2012a, pp. 205-223, http://traces.revues.org/5534. 
Dacos (Marin), « Gratuité ou libre accès ? Poser les termes du débat, c'est déjà y répondre en partie », Blogo-Numericus, 28 octobre 2012b, http://bn.hypotheses.org/11070.

Dacos (Marin) textes réunis par, Read/Write Book. Le livre inscriptible, Marseille, OpenEdition Press, 2010, http://books.openedition.org/oep/128?lang=fr.

Dacos (Marin), « Manifeste des Digital humanities », THATCamp, Paris, 2011, http:// tcp.hypotheses.org/318.

Dacos (Marin), Mounier (Pierre), L'édition électronique, Paris, La Découverte, 2010.

Dardot (Pierre), Laval (Christian), Commun. Essai sur la Révolution au XXIe siècle, Paris, La Découverte, 2014.

Davidson « Cathy », « Drinking the HASTAC Kool-Aid », HASTAC, 2009, http://www.hastac.org/ blogs/cathy-davidson/drinking-hastac-kool-aid.

Déclaration de Berlin sur le Libre Accès à la Connaissance en Sciences exactes, Sciences de la vie, Sciences humaines et sociales, 2003, http://openaccess.mpg.de/68042/

BerlinDeclaration_wsis_fr.pdf

Denouël (Julie), Granjon (Fabien), Aubert (Aurélie), Médias numériques \& participation. Entre engagement citoyen et production de soi, Paris, Mare \& Martin, 2014.

Denouël (Julie), Granjon (Fabien) dir., Communiquer à l'ère numérique. Regards croisés sur la sociologie des usages, Presses des Mines, Paris, 2011.

Dericquebourg (Baptiste), Fondu (Guillaume), Quétier (Jean), « Préface », in Korsch (Karl), Marxisme et philosophie, Paris, Allia, 2012, pp. 7-13.

Dhiparis, «Jeunes chercheurs et humanités numériques : un manifeste », IHA, Paris, 2013, http:// dhiha.hypotheses.org/1108.

Dieter (Michael), «The Virtues of Critical Technical Practices », Differences, vol. 25, n 1, 2014, pp. 216-230.

Differences. A Journal of Feminist Cultural Studies, In the Shadows of the Digital Humanities, vol. 25, $n^{\circ} 1,2014$.

Doueihi (Milad), Ganascia (Jean-Gabriel), « De l'intelligence artificielle aux humanités numériques. Entretien », Laviedesidees.fr, 30 juin 2014, http://www.laviedesidees.fr/De-lintelligence-artificielle-aux.html.

Doueihi (Milad), Qu'est-ce que le numérique ?, Paris, PUF, 2013.

Doueihi (Milad), Pour un humanisme numérique, Paris, Seuil, 2011.

Doueihi (Milad), La grande conversion numérique, Paris, Seuil, 2008.

Eagleton (Terry), Marx et la liberté, Paris, Seuil, 2000.

Ellul (Jacques), La technique ou l'enjeu du siècle, Paris, Économica, 2008.

Epstein (Stephen), Histoire du sida, 2 tomes, Paris, Les Empêcheurs de tourner en rond, 2001.

Faucilhon (Joël), « Portrait du pirate en conservateur de bibliothèque », in Read/Write Book. Le livre inscriptible, Marseille, OpenEdition Press, 2010, http://books.openedition.org/oep/128.

Feenberg (Andrew), Pour une théorie critique de la technique, Montréal, Lux, 2014.

Fitzpatrick (Kathleen), The Humanities, Done Digitally », in Gold (Matthew K.) ed., Debates in the Digital Humanities, Minneapolis, University of Minnesota Press, 2012, pp. 12-15. 
Fitzpatrick (Kathleen), Planned Obsolescence. Publishing, Technology, and the Future of the Academy, New York, NYU Press, 2011a.

Fitzpatrick (Kathleen), « The Humanities, Done Digitally », The chronicle of Higher Education, mai 2011b, http://chronicle.com/article/The-Humanities-Done-Digitally/127382/.

Flanders (Julia), « Time, Labor, and "alternate Careers" in Digital Humanities Knowledge Work », in Gold (Matthew K.) ed., Debates in the Digital Humanities, Minneapolis, University of Minnesota Press, 2012, pp. 292-308.

Fourmentraux (Jean-Paul) dir., L'Ère post-média. Humanités digitales et Cultures numériques, Paris, Hermann, 2012.

Fuchs (Christian), Digital labour and Karl Marx, New York/Londres, Routledge, 2014.

Fuchs (Christian), Social media: a critical introduction, Londres, Sage, 2013.

Fyfe (Paul), « Electronic Errata: Digital Publishing, Open Review, and the Futures of Correction », in Gold (Matthew K.) ed., Debates in the Digital Humanities, Minneapolis, University of Minnesota Press, 2012, pp. 259-280.

Garo (Isabelle), L'Or des images. Art-Monnaie-Capital, Montreuil, La Ville Brûle, 2013.

Gold (Matthew K.) ed., Debates in the Digital Humanities, Minneapolis, University of Minnesota Press, 2012.

Gold (Matthew K.), « The Digital Humanities Moment », in Gold (Matthew K.) ed., Debates in the Digital Humanities, Minneapolis, University of Minnesota Press, 2012, pp. IX-XVI.

Goldmann (Lucien), Sciences humaines et philosophie, Paris, Gonthier, 1966.

Gorz (André), Ecologica, Paris, Galilée, 2007.

Granjon (Fabien), « La critique est-elle soluble dans les sciences de l'information et de la communication? », in George (Éric), Granjon (Fabien) dir., Critique, sciences sociales et communication, Paris, Mare \& Martin, 2014a, pp. 291-355.

Granjon (Fabien), « De la participation médiatique à la participation politique », in Denouël (Julie), Granjon (Fabien), Aubert (Aurélie), Médias numériques \& participation. Entre engagement citoyen et production de soi, Paris, Mare \& Martin, 2014b, pp. 167-185.

Granjon (Fabien) dir., De quoi la critique est-elle le nom ?, Paris, Mare \& Martin, 2013.

Granjon (Fabien), Magis (Christophe), «Vers une "nouvelle anthropologie” critique ? Jalons pour une épistémologie matérialiste des humanités numériques », Journal des anthropologues, n 142-143, 2015, pp. 281-303.

Grusin (Richard), « The Dark Side of the Digital Humanities - Part 2 », Thinking C21, 2013, http:// www.c21uwm.com/2013/01/09/the-dark-side-of-the-digital-humanities-part-2/.

Gunthert (André), « Quelques questions à discuter avec @SebCalvet », 10 octobre 2012, http:// culturevisuelle.org/icones/2536.

Harribey (Jean-Marie), « Le cognitivisme, nouvelle société ou impasse théorique et politique ? ", Actuel Marx, $\mathrm{n}^{\circ}$ 36, 2004, p. 151-180.

Hayles (N. Katherine), « How We Think: Transforming Power and Digital Technologies » in Berry (David M.) ed., Understanding Digital Humanities, New-York, Palgrave Macmillian, 2012, p. 42-66.

Hesmondhalgh (David), The Cultural Industries, London, Sage, 2007. 
Himanen (Pekka), The Hacker Ethic and the Spirit of the Information Age, New York, Random House, 2001.

Hirsch (Brett D.), ed., Digital Humanities Pedagogy. Practices, Principles and Politics, Cambridge, OpenBook Publishers, 2012.

Hooks (Bell), Teaching to Transgress. Education as the Practice of Freedom, New York, Routlege, 1994.

Horkheimer Max, Théorie traditionnelle et théorie critique, Paris, Payot, 1974.

Hui Kyong Chun (Wendy), « The Dark Side of the Digital Humanities - Part 1 », Thinking C21, 2013, http://www.c21uwm.com/2013/01/09/the-dark-side-of-the-digital-humanities-part-1/.

Hui Kyong Chun (Wendy), Rhody (Lisa Marie), « Working the Digital Humanities: Uncovering Shadows between the Dark and the Light », Differences, vol. 25, $\mathrm{n}^{\circ}$ 1, spring 2014, pp. 1-25.

Jakubowsky (Franz), Les superstructures idéologiques dans la conception matérialiste de l'histoire, Paris, EDI, 1971.

Janneke (Adema), « Practise What You Preach: Engaging in Humanities Research through Critical Praxis ", International Journal of Cultural Studies, vol. 16, n 5, 2013, pp. 491-505.

Jones (Steven E.), The emergence of the digital humanities, London, Routledge, 2014.

Kill (Melanie), « Wikipedia, Collaboration, and the Politics of Free Knowledge », in Hirsch (Brett D.) ed., Digital Humanities Pedagogy. Practices, Principeles and Politics, Cambridge, OpenBook Publishers, 2012, pp. 389-406.

Kirschenbaum (Matthew), «What Is Digital Humanities and What's It Doing in English Departments? », in Gold (Matthew K.) ed., Debates in the Digital Humanities, Minneapolis, University of Minnesota Press, 2012, pp. 3-11.

Koh (Adeline), « Niceness, Building, and Opening the Genealogy of the Digital Humanities: Beyond the Social Contract of Humanities Computing ", Differences, vol. 25, n 1, spring 2014, pp. 93-106.

Koh (Adeline), Roopika (Risam), « Humanities as a historical "refuge” from race/class/gender/ sexuality/disability? ", Postcolonial Digital Humanities, 2013, http://dhpoco.org/blog/2013/05/10/ open-thread-the-digital-humanities-as-a-historical-refuge-fromraceclassgendersexualitydisability/.

Korsch (Karl), Au cœur de la conception matérialiste de l'histoire, Paris, Spartacus, 1979.

Kosik (Karel), La crise des temps modernes. Dialectique de la morale, Paris, Les Éditions de la passion, 2003.

Kosik (Karel), La dialectique du concret, Paris, Les Éditions de la passion, 1988.

Kroker (Arthur), Kroker (Marilouise), Critical Digital Studies: A Reader, Toronto, Toronto University Press, 2008.

(de) Lagasnerie (Geoffroy), Logique de la création, Paris, Fayard, 2011.

Lahire (Bernard), Monde pluriel. Penser l'unité des sciences sociales, Paris, Seuil, 2012.

Latour (Bruno), « La fin des moyens », Réseaux, vol. 18, n 100, 2000, pp. 38-58.

Le Crosnier (Hervé), « Le libre accès à la connaissance, à la culture, aux émotions et à

l'élaboration collective ", in Peugeot (Valérie) dir., Pouvoir Savoir, Caen, C\&F, p. 219-224. 
Lessig (Lawrence), «Industries de la culture, pirates et "culture libre” », Critique, n 733-734, 2008, pp. 510-518.

Liu (Alan), « Where Is Cultural Criticism in the Digital Humanities? », in Gold (Matthew K.) ed., Debates in the Digital Humanities, Minneapolis, University of Minnesota Press, 2012, pp. 490-509.

Losh (Elizabeth), « Hacktivism and the Humanities: Programming Protest in the Era of the Digital University ", in Gold (Matthew K.) ed., Debates in the Digital Humanities, Minneapolis, University of Minnesota Press, 2012, pp. 161-186.

McPherson (Tara), « Why Are the Digital Humanities so White? Or Thinking the Histories of Race and Computation », in Gold (Matthew K.) ed., Debates in the Digital Humanities, Minneapolis, University of Minnesota Press, 2012, pp. 139-160.

McPherson (Tara), «Introduction: Media Studies and the Digital Humanities », Cinema Journal, vol. 48, n², 2009, pp. 119-123.

Mahony (Silon), Tiedau (Ulrich), Sirmons (Irish), « Open access and online teachnig materials for digital humanities », in Warwick (Claire), Terras (Melissa), Nyhan (Julianne) eds., Digital Humanities in Practice, Londres, Facet Publishing, 2012, pp. 167-191.

Manovich (Lev), « Trending: The Promise and the Challenges of Big Social Data », in Gold (Matthew K.) ed., Debates in the Digital Humanities, Minneapolis, University of Minnesota Press, 2012, pp. 460-475.

Mattelart (Armand), Communication, cultures populaires et émancipation. Une anthologie des écrits d'Armand Mattelart (1970-1986). Tome 2, édition établie et présentée par Fabien Granjon et Michel Sénécal, Paris, Presses des Mines, 2015, à paraître.

Mauss (Marcel), Sociologie et anthropologie, Paris, PUF, 1973.

Moulier-Boutang (Yann), Le capitalisme cognitif, Paris, Amsterdam, 2007.

Mounier (Pierre), « Une introduction aux humanités numériques », in Stiegler (Bernard) dir., Digital Studies. Organologie des savoirs et technologies de la connaissance, Paris, IRI/FYP, 2014a, pp. 95-108.

Mounier (Pierre), « Histoire et numérique : une relation singulière et multiple », Homo numericus, 2014b, http://www.homo-numericus.net/article314.html.

Mounier (Pierre), « Avant-propos », in Mounier (Pierre) dir., Read/Write Book 2. Une introduction aux humanités numériques, Marseille, OpenEdition Press, 2012, http://books.openedition.org/ oep/241.

Mounier (Pierre), «Qu'apportent les digital humanities ? Quelques exemples », Homo numericus, 2011, http://www.homo-numericus.net/breve1011.html.

Mounier (Pierre), «L'édition électronique : un nouvel eldorado pour les sciences humaines ?», in Dacos (Marin), Read/Write Book. Le livre inscriptible, Marseille, OpenEdition Press, 2010, http:// books.openedition.org/oep/169.

Murray-John (Patrick), « Theory, Digital Humanities, and Noticing », Journal of Digital Humanities, vol. 1, n 1, 2011, http://journalofdigitalhumanities.org/1-1/theory-digital-humanities-andnoticing-by-patrick-murray-john/.

Negri (Antonio), Vercellone (Carlo), « Le rapport capital/travail dans le capitalisme cognitif », Multitudes, $n^{\circ} 32,2008$, pp. 39-50. 
Noyer (Jean-Max), « Connaissance, pensée, réseaux à l'heure numérique. Pour une nouvelle Renaissance »,Les Cahiers du numérique, vol. 6, n³, 2010, pp. 187-209.

Oliver (Julian), Savicic (Gordan), Vasiliev (Danja), « The Critical Engineering Manifesto », 2011, http://criticalengineering.org/fr.

Ostrom (Elinor), Governing the Commons. The Evolution of Institutions for Collective Action, Cambridge UK, Cambridge University Press, 1990.

Ostrom (Elinor), Hess (Catherine) dir., Understanding Knowledge as Commons, Cambridge (MA), MIT Press, 2007.

Ouakrat (Alan), Mésengeau (Julien), « Resocialiser les traces d'activités numériques : une proposition qualitative pour les SIC ", Revue française des sciences de l'information et de la communication, $\mathrm{n}^{\circ} 8,2016$, https://rfsic.revues.org/1795.

Peugeot (Valérie), « Les biens communs, une utopie pragmatique », in Vecam (ed.), Libres savoirs. Les biens communs de la connaissance, Caen, C\&F, 2011.

Pitti (Daniel V.), « Designing Sustainable Projects and Publications », in Schreibman (Susan), Siemens (Ray), Unsworth (John) eds., A companion to Digital Humanities, Oxford, Blackwell Publishing, 2004, pp. 471-487.

Raley (Rita), « Digital Humanities for the Next Five Minutes », Differences, vol. 25, n 1, spring 2014, pp. 1-25.

Ramsay (Stephen), « Who's In and Who's Out? », 2011, http://stephenramsay.us/ text/2011/01/08/whos-in-and-whos-out/.

Ramsay (Stephen), Rockwell (Geoffroy), « Developping Things: Notes toward an Epistemology of Building in the Digital Humanities », in Gold (Matthew K.) ed., Debates in the Digital Humanities, Minneapolis, University of Minnesota Press, 2012, pp. 75-84.

Reber (Bernard), Brossaud (Claire), « Conclusion », in Brossaud (Claire), Reber (Bernard), Humanités numériques 1. Nouvelles technologies cognitives et épistémologies, Paris, Hermes/Lavoisier, 2007, pp. 269-276.

Renaud (Pascal), « Formation, logiciels libres et partenariat public-privé », in Peugeot (Valérie) dir., Pouvoir Savoir, Caen, C\&F, p. 225-234.

Réseaux, « Trente années d'une revue », vol. 32, n 184-185, 2014.

Schmidt (Benjamin M.), « Theory First », Journal of Digital Humanities, vol. 1, n 1, 2011, http:// journalofdigitalhumanities.org/1-1/theory-first-by-ben-schmidt/.

Schnapp (Jeffrey), Lunenfeld (Peter), Presner (Todd), " The Digital Humanities Manifesto 2.0 ", UCLA, Los Angeles, 2009, http://manifesto.humanities.ucla.edu/2009/05/29/the-digitalhumanities-manifesto-20/.

Schnapp (Jeffrey), Lunenfeld (Peter), Presner (Todd), « A digital Humanities Manifesto », UCLA, Los Angeles, 2008, http://manifesto.humanities.ucla.edu/2008/12/15/digital-humanitiesmanifesto/.

Schreibman (Susan), Siemens (Ray), Unsworth (John) eds., A companion to Digital Humanities, Oxford, Blackwell Publishing, 2004.

Scheinfeldt (Tom), « Sunset for Ideology, Sunrise for Methodology? », in Gold (Matthew K.) ed., Debates in the Digital Humanities, Minneapolis, University of Minnesota Press, 2012, pp. 125-126. 
Scheinfeldt (Tom), «Stuff Digital Humanists Like: Defining Digital Humanities by its Values », Found History, 2010, http://www.foundhistory.org/2010/12/02/stuff-digital-humanists-like/.

Scheinfeldt (Tom), « Why Digital Humanities is “Nice” », Found History, 2009, http:// www.foundhistory.org/2010/05/26/why-digital-humanities-is-"nice"/.

Silver (David), Massanari (Adrienne) eds., Critical Cyberculture Studies, New York, New York University Press, 2006.

Simondon (Gilbert), Du mode d'existence des objets techniques, Paris, Aubier, 2012.

Smith (Kevin), « The unexpected reader », Scholarly Communications @ Duke, 15 novembre 2011, http://blogs.library.duke.edu/scholcomm/2011/11/15/the-unexpected-reader/.

Spiro (Lisa), « Opening up Digital Humanities Education », in Hirsch (Bret D.) ed., Digital Humanities Pedagogy. Practices, Principeles and Politics, Cambridge, OpenBook Publishers, 2012a, pp. 331-363.

Spiro (Lisa), " "This Is Why We Fight”: Defining the Values of the Digital Humanities ", in Gold (Matthew K.) ed., Debates in the Digital Humanities, Minneapolis, University of Minnesota Press, 2012b, pp. 16-35.

Svensson (Patrik), «The Landscape of Digital Humanities », Digital Humanities Quaterly, vol. 4, $\mathrm{n}^{\circ} 1$, 2010, http://digitalhumanities.org/dhq/vol/4/1/000080/000080.html.

Terras (Melissa), «Un regard jeté sous le chapiteau : les humanités numériques et la crise de l'inclusion ", in Mounier (Pierre) dir., Read/Write Book 2. Une introduction aux humanités numériques, Marseille, OpenEdition Press, 2012, http://books.openedition.org/oep/251.

Thatcamp Paris 2012, Non-actes de la non-conférence des humanités numériques, Paris, Éditions de la Maison des sciences de l'homme, 2012, http://books.openedition.org/editionsmsh/278.

Thibault (Françoise), « De l'université virtuelle au campus numérique : simple effet de traduction ou changement de paradigme », Premier colloque franco-mexicain, Mexico, 8-10 avril 2002, http://edutice.archives-ouvertes.fr/docs/00/00/15/86/PDF/campus.pdf

Unsworth (John), « The Humanist: “Dances With Wolves” or "Bowls Alone”? », 2003, http:// www.arl.org/storage/documents/publications/scholarly-tribes-unsworth-17oct03.pdf

Vecam (ed.), Libres savoirs. Les biens communs de la connaissance, Caen, C\&F, 2011.

Vecam (ed.), Pouvoir Savoir. Le développement face aux biens communs de l'information et à la propriété intellectuelle, Caen, C\&F, 2005.

Vercellone (Carlo) dir., Sommes-nous sortis du capitalisme industriel ?, Paris, La Dispute, 2003.

Vinck (Dominique), Pratiques de l'interdisciplinarité. Mutation des sciences, de l'industrie et de l'enseignement, Grenoble, PUG, 2000.

Voirol (Olivier), « Matérialisme interdisciplinaire et critique de la culture », in Noppen (PierreFrançois), Raulet (Gérard), Macdonald (Iain) dir., les Normes et le possible. Héritage et perspectives de l'École de Francfort, Paris, Éditions de la maison des sciences de l'homme, 2012, pp. 19-51.

Warwick (Claire), Terras (Melissa), Nyhan (Julianne) eds., Digital Humanities in Practice, Londres, Facet Publishing, 2012.

Wieviorka (Michel), L'impératif numérique ou La nouvel ère des sciences humaines et sociales ?, Paris, CNRS Éditions, 2013.

Wharton (Robin), « Digital Humanities, Copyright Law, and the Literary », 2013, http:// www.digitalhumanities.org/dhq/vol/7/1/000147/000147.html. 
Whitson (Roger), « On DH, Solidarity, and Humility - or Why @miriamkp Rules », 2012, http:// www.rogerwhitson.net/?p=1376.

\section{NOTES DE FIN}

1. L'expression « humanités numériques » $(\mathrm{HN})$ sera souvent utilisée dans la suite du texte comme un hyperonyme ne désignant finalement qu'une partie de ce vaste territoire que sont lesdites « humanités numériques ».

2. Une version courte de ce papier a été publiée dans le $\mathrm{n}^{\circ} 142-143$ du Journal des anthropologues : «Vers une "nouvelle anthropologie" critique ? Jalons pour une épistémologie matérialiste des humanités numériques » (2015 : 281-303).

3. «The dark side of digital humanities » est, à l'origine, le titre donné à une table ronde organisée par Richard Grusin à la convention 2013 de la Modern Language Association (MLA) et dont la question introductive était : «Est-ce seulement un accident si l'émergence des humanités numériques a coïncidé avec l'intensification de la crise économique dans les sciences humaines au sein de l'enseignement supérieur, ou existe-t-il un lien entre ces deux faits? ». L'expression entend ainsi désigner les limites et les différents types de travers propres aux digital humanities (déterminisme technique, précariat, manque d'intérêt pour les approches genrées, etc.) et par glissement, elle signifie également la critique même (le travail du négatif) de cette «face cachée » (Differences, 2014).

4. Cet élan critique n'est pas sans contradiction et se nourrit également d'impératifs libéraux qui pèsent sur la sphère académique et enjoignent certaines humanités « en crise » (Mounier, 2014b) à s'appuyer sur le numérique pour se redonner quelque couleur (celle de la modernité) : " Ajouter le qualificatif “numérique" aux humanités peut sembler être, pour certains, la nouvelle manière d'étayer les Arts et Humanités lors de la dernière version d'une longue crise dans l'enseignement supérieur, et cet étayage n'est pas sans implications sur la précarisation croissante du travail académique. Les praticiens des Humanités numériques que je connais sont bien conscients de ces complications et de ces complicités et ils se retrouvent souvent à devoir répondre, tempérer et résister à de telles vues un peu simplistes » (Jones, $2014: 10$ ).

5. Nous reviendrons, dans la contribution suivante, sur la valorisation de la figure de cet unexpected reader. Il convient tout de même ici de préciser qu'elle est véhiculée avec tous les éléments typiques des constructions de figures mythiques. En effet, dans le texte original de Kevin Smith (2011), il s'agit d'une jeune élève de lycée de quinze ans qui, en lisant des articles spécialisés en biologie, via des accès « test » à des catalogues scientifiques, aurait fini par écrire un article à son tour, pour le proposer au rédacteur en chef de la revue PLoS Computational Biology. Ce dernier, attestant de l'innovation majeure proposée par l'article l'a finalement publié, non sans l'avoir préalablement proposé à la prestigieuse revue Science.

6. Le cas de la recherche sur le sida est à cet égard exemplaire (Epstein, 2001).

7. Un exemple paradigmatique de cet ajustement étant probablement, en France, le colloque « Propriété littéraire et artistique et Humanités numériques » dont le programme annonce s'intéresser aux questions de propriété intellectuelle afin notamment de savoir « comment constituer des corpus numériques sans porter atteinte aux droits de propriété littéraire et artistique [et] exploiter ces corpus en respectant les droits qui y sont attachés » : http://humanitesnumeriques.wordpress.com.

8. Cf. http://openaccess.mpg.de/68042/BerlinDeclaration_wsis_fr.pdf. 
9. Ainsi par exemple lorsqu'André Gunthert affirme que « l'un des enjeux primordiaux de l'économie de demain est de réussir à articuler commerce et partage, plutôt que de les opposer comme des ennemis irréductibles » (Gunthert, 2012).

10. En économie traditionnelle, un bien non-exclusif est un bien dont tout le monde peut bénéficier une fois produit, c'est-a-dire pour lequel il est très difficile, voire impossible d'en restreindre l'accès. Le caractère rival d'un bien signifie que sa consommation par un agent économique l'expose à une réduction de sa quantité ou de sa qualité disponibles pour les autres agents.

11. Pour un florilège de définitions des humanités numériques, cf. les réponses apportées à la question «Comment définissez-vous les digital humanities? ", posée aux participants du rassemblement Day of DH 2012 : http://dayofdh2012.artsrn.ualberta.ca/dh/.

12. Citant les travaux d'Antonio Casilli et Paola Tubaro (2011) sur le rôle de la téléphonie mobile et des médias sociaux dans les révoltes en Grande-Bretagne de 2011, Wieviorka affirme que ces chercheurs montrent que « si la communication est coupée [...], l'émeute se prolonge beaucoup plus ; les réseaux sociaux et les messageries instantanées joueraient donc un rôle plutôt positif, en limitant l'étendue et la durée de l'émeute ». Et d'ajouter qu'il s'agit là d'une recherche qui, par la mise en œuvre d'outils de « simulation sociale » (des agents de Epstein - cf. la contribution d'Arthur Lefèvre dans le présent dossier), aurait pour avantage de «mettre fin à un préjugé politiquement manipulé » : celui qui présente les médias numériques comme catalyseurs des émeutes (Wieviorka, 2013 : 46). Ce serait par ailleurs un exemple d'engagement pratique typique d'une forme de " just in time sociology » dont les résultats, publicisés durant l'événement même dont l'article traite, et en dehors du circuit académique, permettraient de répondre avec les armes des SHS à l'urgence d'une situation sociale et politique inquiétante. L'article démontrerait ainsi « que les sciences sociales peuvent éclairer l'action politique sur un point précis au moment où elle en a besoin, et, en utilisant les mêmes moyens de communication qu'elle, participer en temps réel au débat public » (Mounier, 2011). Mais ne se place-t-on pas, là, sous les auspices utilitaristes du problem solving que nous évoquions supra? Outre le fait qu'il serait utile de se poser la question de ce que cette méthode doit à une vision quelque peu réductionniste et fonctionnaliste de type « engineering social », il serait tout aussi salutaire de se pencher sur la portée normative de la conclusion dudit article, laquelle tend à délégitimer le type de conflictualité sociale étudié. En l'occurrence, la " critique » sous-jacente à ce type d'approche vise moins à expliciter ce que ce type de manifestation agonistique doit par exemple aux conditions sociales d'existence des révoltés, et ainsi mettre en regard le fait étudié avec un ordre social, qu'à « susciter un débat public et la participation politique » (Casilli, Tubaro, 2011) : une activité à laquelle les auteurs prennent part en suggérant que la censure des médias sociaux est une mauvaise chose dans la mesure où elle remet en cause la liberté d'expression, mais aussi parce qu'elle ne contribue pas à « pacifier les sociétés rebelles ", c'est-à-dire conduit à créer des dysfonctionnements sociétaux.

\section{INDEX}

Mots-clés : humanité numérique, critique, interdisciplinarité, praxis, totalisation 


\section{AUTEURS}

\section{FABIEN GRANJON}

Professeur en Sciences de l'information et de la communication, Université Paris 8 - Cemti

\section{CHRISTOPHE MAGIS}

Maître de Conférences en Sciences de l'information et de la communication, Université Paris 8 Cemti 